Research Article

\title{
Porous Multiphase Microcharacteristics Research of New Cement-Based Grouting Material Concretion
}

\author{
Wei Xie $\mathbb{D}^{1,2}$ Junwu Xia $\mathbb{D}^{1,3}$ Linli Yu $\mathbb{D}^{1},{ }^{1}$ and Hongfei Chang $\mathbb{D}^{1,3}$ \\ ${ }^{1}$ School of Mechanics and Civil Engineering, China University of Mining and Technology, Xuzhou 221116, China \\ ${ }^{2}$ Xuhai College, China University of Mining and Technology, Xuzhou 221008, China \\ ${ }^{3}$ Jiangsu Key Laboratory of Environmental Impact and Structural Safety in Engineering, Xuzhou 221116, China
}

Correspondence should be addressed to Junwu Xia; xjunw@163.com

Received 26 September 2020; Revised 9 November 2020; Accepted 21 December 2020; Published 9 January 2021

Academic Editor: Xun Xi

Copyright ( $(2021$ Wei Xie et al. This is an open access article distributed under the Creative Commons Attribution License, which permits unrestricted use, distribution, and reproduction in any medium, provided the original work is properly cited.

\begin{abstract}
As an excellent inorganic hydraulic cementitious material, new cement-based grouting material (abbreviated as NCG) has a wide application prospect in grouting engineering. Four types of water-cement ratio (W/C ratio) NCG concretions were systematically researched on the solid-phase composition, microscopic morphology, nanoscale pore characteristics and micron-scale multiphase microscopic characteristics through SEM, TGA-DSC, BET, MIP, and 3D-XRM in combination with hydration reaction mechanism. The NCG concretion is characterized as a spatial network structure formed by dendritic or columnar ettringite (AFt) crystals, filled with calcium silicate hydrate gel $(\mathrm{C}-\mathrm{S}-\mathrm{H})$ and aluminum gel $\left(\mathrm{AH}_{3}\right)$. In the solid phase, the mass of $\mathrm{AFt}$ is the highest, about $65 \%$, the mass of $\mathrm{C}-\mathrm{S}-\mathrm{H}$ is about $20 \%$, and the mass of $\mathrm{AH}_{3}$ is the least, about $15 \%$. In nanoscale, the pore size of NCG concretion is concentrated in about $50 \mathrm{~nm}-3 \mu \mathrm{m}$, and the volume proportion reaches about $90 \%$, which can be used as the characteristic parameter of pore-phase. With the increase of W/C ratio and age, the MPD of pore decreases, and the probability of the MPD-pore increases, and the dispersion of the probability of pores increases. In nanoscale, the porosity of NCG concretion is as high as $60 \%$. With the increase of W/C ratio and age, the porosity of NCG concretion increases, but the effect on the density of solid-phase is little. In micron scale, the regular characteristics related to diameter of pore-phase and gel-phase are similar, showing a three stage formula: as their diameter increases, the number of monocases decreases and its rate of reduction gradually slows down, the volume-proportion decreases first and then increases, the differentiation of volume between the monocases increases gradually, and the differentiation between monocases shape and sphere is bigger, and its shape characteristics are more diverse and discrete.
\end{abstract}

\section{Introduction}

Grouting has been widely used in various fields, such as mining, water conservancy, underground, construction, road, and bridge. The properties and types of grouting materials determine the construction technology and application scene. After more than 200 years of development, there are various types of grouting slurry, including three major categories: cement slurry (e.g., single cement slurry, cement-sodium silicate slurry, clay cement slurry, etc.), chemical slurry (e.g., sodium silicate, propylene phthalimide, lignin, epoxy resin, chrome lignin, formaldehyde resin, polyurethane, etc.), and composite cement-chemical slurry $[1-4]$.
A new cement-based grouting material (hereinafter referred to as NCG) was studied in this paper, belonging to the high water-cement ratio (volumetric moisture content is $80 \%-90 \%)$ inorganic hydraulic gel material. It consists of material A (usually composed of CA, CAS, or CAF cement sintering, suspension concentrate, dispersant, and retarder) and material B (usually composed of gypsum, lime, suspension concentrate, dispersant, and accelerator). Material $\mathrm{A}$ and material B are separately added with water to form a single slurry, which has the advantages of long time nonsetting, high fluidity, good pumpability, and no pipe plugging. After mixing two single parts, the grouting material can quickly set and harden to reach early strength, with excellent performance. The grouting material was applied widely to 
solve engineering problems such as geological cavity, fracture, and broken rock caused by the production activities in the fields of construction, water conservancy, transportation, mining, metallurgy, environmental protection, and petroleum. Many scholars have studied the hydration reaction mechanism [5-7] and physical properties [8-10] of this kind of cement-based grouting material, and the microscopic structure characteristics of the concretion are involved in the research of mechanism and performance.

Apparently, the microstructure of the concretion of cement-based material determines its macroscopic performance. In the study of macro performances of materials such as strength, corrosion, and cracking [11-13], more and more attention has been paid to microscale and microcharacteristics. In view of the new cement-based grouting material, combined with the hydration reaction mechanism, a comprehensive and systematic study on the solid composition, micro morphology, nanoscale pore characteristics and micron-scale multiphase characteristics is carried out by using a variety of test and analysis methods, which are of significance for understanding the macro physical properties of concretion and mechanical behavior of porous multiphase media.

\section{NCG Material and Specimens}

The composition of NCG is shown in Figure 1.

By different mix proportions, the setting time, strength, and other physical properties of NCG materials can be adjusted to form different NCG materials. In addition, the $\mathrm{W} / \mathrm{C}$ ratio (the mass ratio of water to solid material) is the key parameter to the material mix formulations. In this paper, three types of specimen are studied, and their identifiers and mix proportions are shown in Table 1. Figure 2 shows the single slurry stirring state and setting hardening concretion of NCG material.

\section{Micromorphology and Solid-Phase- Composition of NCG Concretion}

3.1. Solid-Phase Product of NCG Concretion. Based on the research results [5-7], the hydration reaction of NCG material conforms to the main hydration reaction of CAS cement: $\mathrm{C}_{4} \mathrm{~A}_{3} \overline{\mathrm{S}}-\mathrm{C} \overline{\mathrm{S}}-\mathrm{CaO}-\mathrm{H}_{2} \mathrm{O}$ system. According to the proportion of CAS cement clinker, gypsum, and lime, there are four different hydration reactions (a simplified nomenclature of cement is adopted; that is, $\mathrm{C}=\mathrm{CaO}, \mathrm{S}=\mathrm{SiO}_{2}$, $\mathrm{A}=\mathrm{Al}_{2} \mathrm{O}_{3}, \overline{\mathrm{S}}=\mathrm{SO}_{3}$, and $\mathrm{H}=\mathrm{H}_{2} \mathrm{O}$ ):

$$
\begin{array}{r}
\mathrm{C}_{4} \mathrm{~A}_{3} \overline{\mathrm{S}}+18 \mathrm{H} \longrightarrow \mathrm{C}_{3} \mathrm{AS}_{3} \mathrm{H}_{12}+2 \mathrm{AH}_{3} \\
3 \mathrm{C}_{4} \mathrm{~A}_{3} \overline{\mathrm{S}}+2 \mathrm{C} \overline{\mathrm{S}} \mathrm{H}_{2}+34 \mathrm{H} \longrightarrow 3 \mathrm{C}_{6} \mathrm{~A}_{3} \mathrm{H}_{32}+4 \mathrm{AH}_{3} \\
2 \mathrm{CS}+2 \mathrm{H} \longrightarrow \mathrm{C}-\mathrm{S}-\mathrm{H}+\mathrm{CH} \\
\mathrm{C}_{4} \mathrm{~A}_{3} \overline{\mathrm{S}}+8 \mathrm{C} \overline{\mathrm{S}} \mathrm{H}_{2}+6 \mathrm{CH}+74 \mathrm{H} \longrightarrow 3 \mathrm{C}_{6} \mathrm{~A}_{3} \mathrm{H}_{32}
\end{array}
$$

Therefore, solid-phase products of NCG mainly include ettringite $\left(\mathrm{C}_{6} \mathrm{~A} \overline{\mathrm{S}}_{3} \mathrm{H}_{32}\right.$, referred to as $\left.\mathrm{AFt}\right)$, aluminum gel $\left(\mathrm{AH}_{3}\right)$, and hydrated calcium silicate gel (shortened to $\mathrm{C}-\mathrm{S}-\mathrm{H})$. The ettringite $(\mathrm{AFt})$ is a trigonal system with needle-columnar crystal structure and the aluminum gel has mainly indefinite shape, and the morphology of hydrated calcium silicate gel $(\mathrm{C}-\mathrm{S}-\mathrm{H})$ is more diverse, mainly in thin fragments, fibrous or lamellae $[14,15]$.

SEM (scanning electron microscope) and TGA-DSC (thermogravimetry-differential scanning calorimetry) were used to study the microscopic characteristics and solidphase composition of NCG concretion in this paper.

3.2. Microscopic Morphology SEM Analysis of NCG Concretion. The high-power microscopic morphology of W1 N, W2 N, and W4 N concretions is obtained by SEM test, as shown in Figure 3.

Based on the prediction of the products of hydration reaction and description of the morphology of AFt and C-S-H gel $[14,15]$, SEM analysis of different NCG concretions shows that the solid-phases in the concretion are mainly ettringite $(\mathrm{AFt})$, aluminum gel $\left(\mathrm{AH}_{3}\right)$, and hydrated calcium silicate gel $(\mathrm{C}-\mathrm{S}-\mathrm{H})$, presenting a typical spatial network structure characteristic. The needle-prismatic AFt crystal interlaces form a spatial network structure, which accounts for the most. In addition, $\mathrm{AH}_{3}$ and $\mathrm{C}-\mathrm{S}-\mathrm{H}$ are filled between intervals of AFt crystal. With the increase of W/C ratio, $\mathrm{AFt}$ crystal becomes thinner and shorter, and the density and regularity of network structure also decline.

3.3. TGA-DSC Analysis on Solid-Phase Composition of NCG Concretion. By the combination of TGA (thermogravimetry analysis) and DSC (differential scanning calorimetry), the possible reaction process of material can be judged from the endothermic peak and exothermic peak of DSC curve and the corresponding TGA curve with mass loss or not, so as to determine the property of the transition peak. TGA-DSC was used to analyze the $\mathrm{W} 2 \mathrm{~N}$ concretion in this paper. Before the test, NCG concretion specimens were immersed in ethyl ethanol (7 days), dried at a constant temperature $\left(60^{\circ} \mathrm{C}\right)$, and ground (less than 200 mesh) for powder to stop the hydration and reduce the influence of adsorbed water. The TG curve, DTG curve, and DSC curve of the sample were obtained by heating from room temperature at the rate of $5^{\circ} \mathrm{C} / \mathrm{min}$ to $1000^{\circ} \mathrm{C}$ under the protection of nitrogen atmosphere, as shown in Figure 4. Among them, the TG curve shows the relationship between mass and temperature, reflecting the stage, trend, and variable quantity of mass change; DTG curve is the result of first-order differential of TG curve, reflecting the rate relationship between mass and temperature; DSC curve exhibits the relationship between heat of the substance and temperature, which is the power output difference of them under programmatic heating (or cooling) at the same temperature.

According to the DSC curve in Figure 4, three heat absorption peaks of the NCG concretion appear at $90^{\circ} \mathrm{C}$, $230^{\circ} \mathrm{C}$, and $670^{\circ} \mathrm{C}$, respectively, corresponding to the hydrolysis of $\mathrm{AFt}$, the dehydration of $\mathrm{AH}_{3}$, and the dehydration of hydrated calcium silicate gel (C-S-H) [16-19], which consist of three extremums of DTG curve, respectively, indicating the extreme value of mass change rate. The temperature returns to normal level after the extreme point 


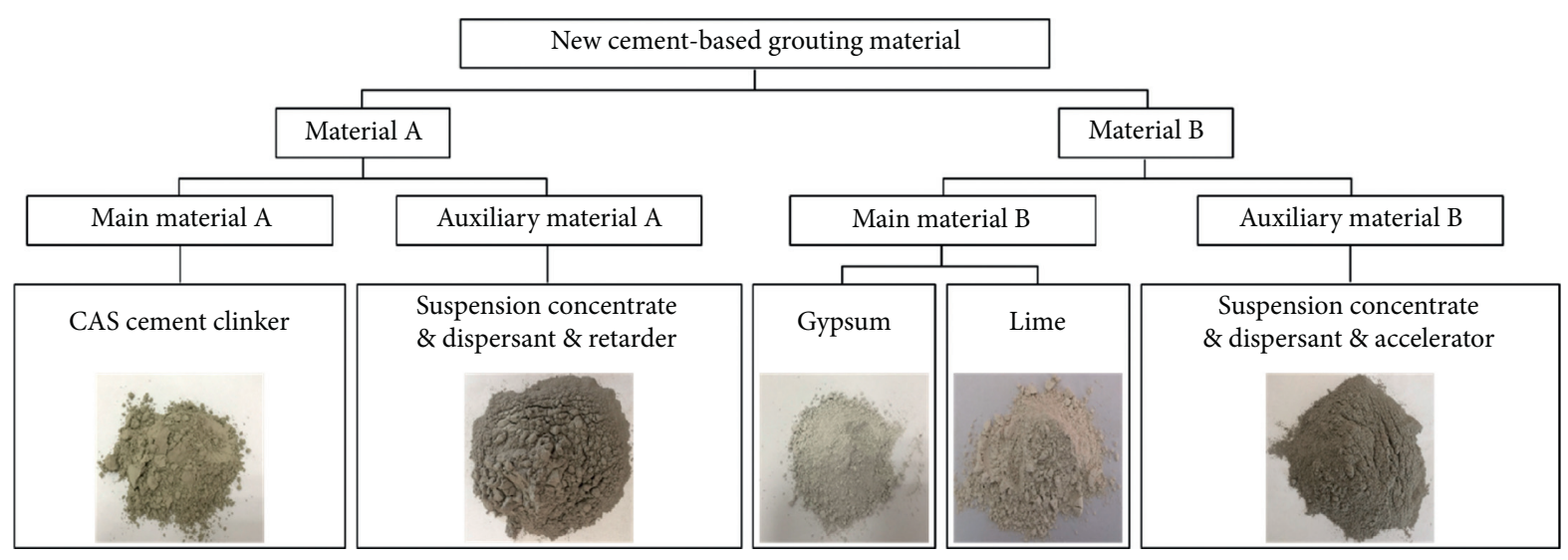

FIGURE 1: Raw material component of NCG material.

TABLE 1: Mix proportions of NCG specimens.

\begin{tabular}{|c|c|c|c|c|c|c|c|c|}
\hline \multirow[b]{2}{*}{ Identifier } & \multirow[b]{2}{*}{$\mathrm{W} / \mathrm{C}$ ratio } & \multicolumn{3}{|c|}{ Material A/(g) } & \multicolumn{4}{|c|}{ Material B/(g) } \\
\hline & & $\begin{array}{c}\text { Main material A } \\
\text { CAS cement clinker }\end{array}$ & Auxiliary material A & Water & $\begin{array}{l}\text { Main mat } \\
\text { Gypsum }\end{array}$ & $\begin{array}{l}\text { rial B } \\
\text { Lime }\end{array}$ & Auxiliary material B & Water \\
\hline W1 N & 1 & 100 & 10 & 110 & 80 & 20 & 10 & 110 \\
\hline W2 N & 2 & 100 & 10 & 220 & 80 & 20 & 10 & 220 \\
\hline W4 N & 4 & 100 & 10 & 440 & 80 & 20 & 10 & 440 \\
\hline
\end{tabular}

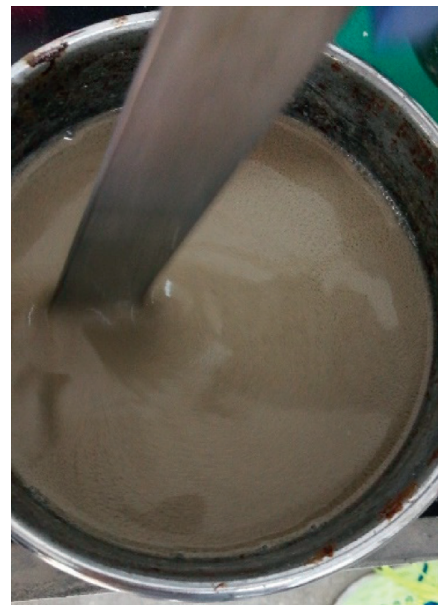

(a)

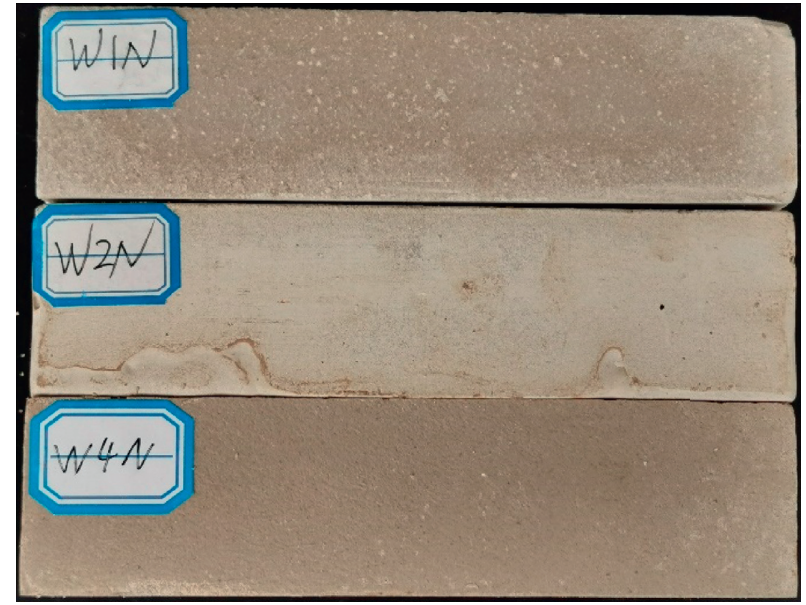

(b)

Figure 2: Preparation of NCG concretion. (a) Single slurry stirring state. (b) Setting hardening concretion.

in DTG curve, at which the corresponding three solid-phase hydrolysis (or dehydration) processes end. Based on the TG curve, the corresponding solid-phase composition of NCG concretion hydrolyzes or dehydrates, leading to the mass reduction.

Combined with the related studies [16-19] and TG curve, the results can be obtained: (1) At about $90^{\circ} \mathrm{C}, \mathrm{AFt}$ $\left(3 \mathrm{CaO} \cdot \mathrm{Al}_{2} \mathrm{O}_{3} \cdot 3 \mathrm{CaSO}_{4} \cdot 32 \mathrm{H}_{2} \mathrm{O}\right)$ lost 14 crystalline water [20], resulting in a mass reduction of $17.5 \%$. (2) At about $230^{\circ} \mathrm{C}$, $\mathrm{AH}_{3}\left(\mathrm{Al}_{2} \mathrm{O}_{3} \cdot 3 \mathrm{H}_{2} \mathrm{O}\right)$ lost 3 crystalline water and transformed to $\mathrm{Al}_{2} \mathrm{O}_{3}$ with mass reduction of $7.6 \%$. (3) At about $670^{\circ} \mathrm{C}$, the dehydration of $\mathrm{C}-\mathrm{S}-\mathrm{H}\left(\mathrm{CaO} \cdot 2 \mathrm{SiO}_{2} \cdot 3 \mathrm{H}_{2} \mathrm{O}\right)$ lost 3 crystalline water, resulting in a $6.1 \%$ reduction in mass (note: the basic composition of $\mathrm{C}-\mathrm{S}-\mathrm{H}$ is $x \mathrm{CaO} \cdot y \mathrm{SiO}_{2} \cdot z \mathrm{H}_{2} \mathrm{O}$, which has a wide range of chemical composition. Considering the hydration reaction characteristics of NCG material and the small proportion of $\mathrm{C}-\mathrm{S}-\mathrm{H}$, it is reasonable to assume that it is $\left.\mathrm{CaO} \cdot 2 \mathrm{SiO}_{2} \cdot 3 \mathrm{H}_{2} \mathrm{O}\right)$.

Considering the mass reduction proportion caused by the molecular formula and water loss amount of solid-phase and the error caused by water loss and adsorbed water of other solid-phases, the total mass proportion of three kinds of solid-phases is corrected with the target of $100 \%$. The mass proportion of three main solid-phases components can be approximately obtained from formulas (2) and (3), as shown in Figure 5. 


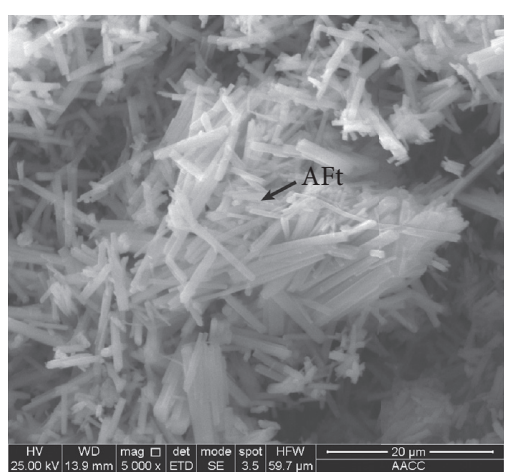

(a)

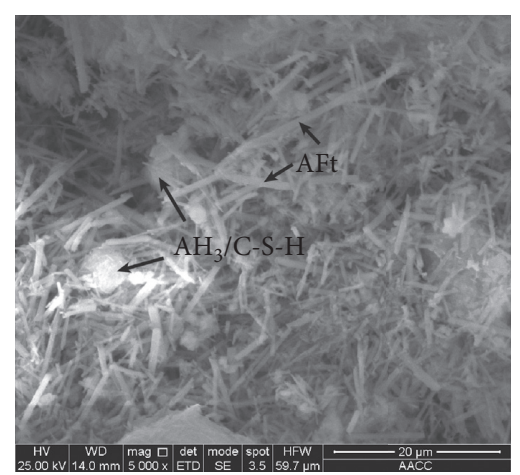

(b)

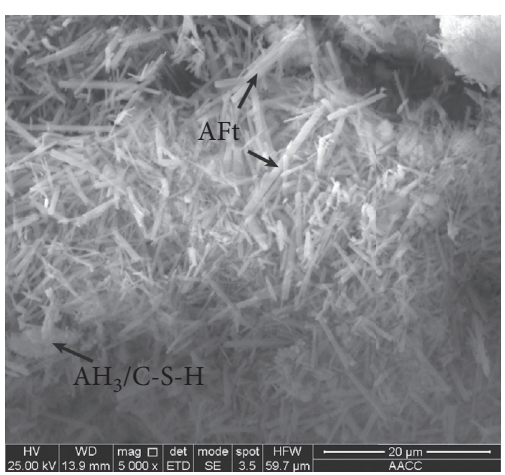

(c)

Figure 3: Micromorphology of NCG concretion. (a) W1 N. (b) W2 N. (c) W4 N.

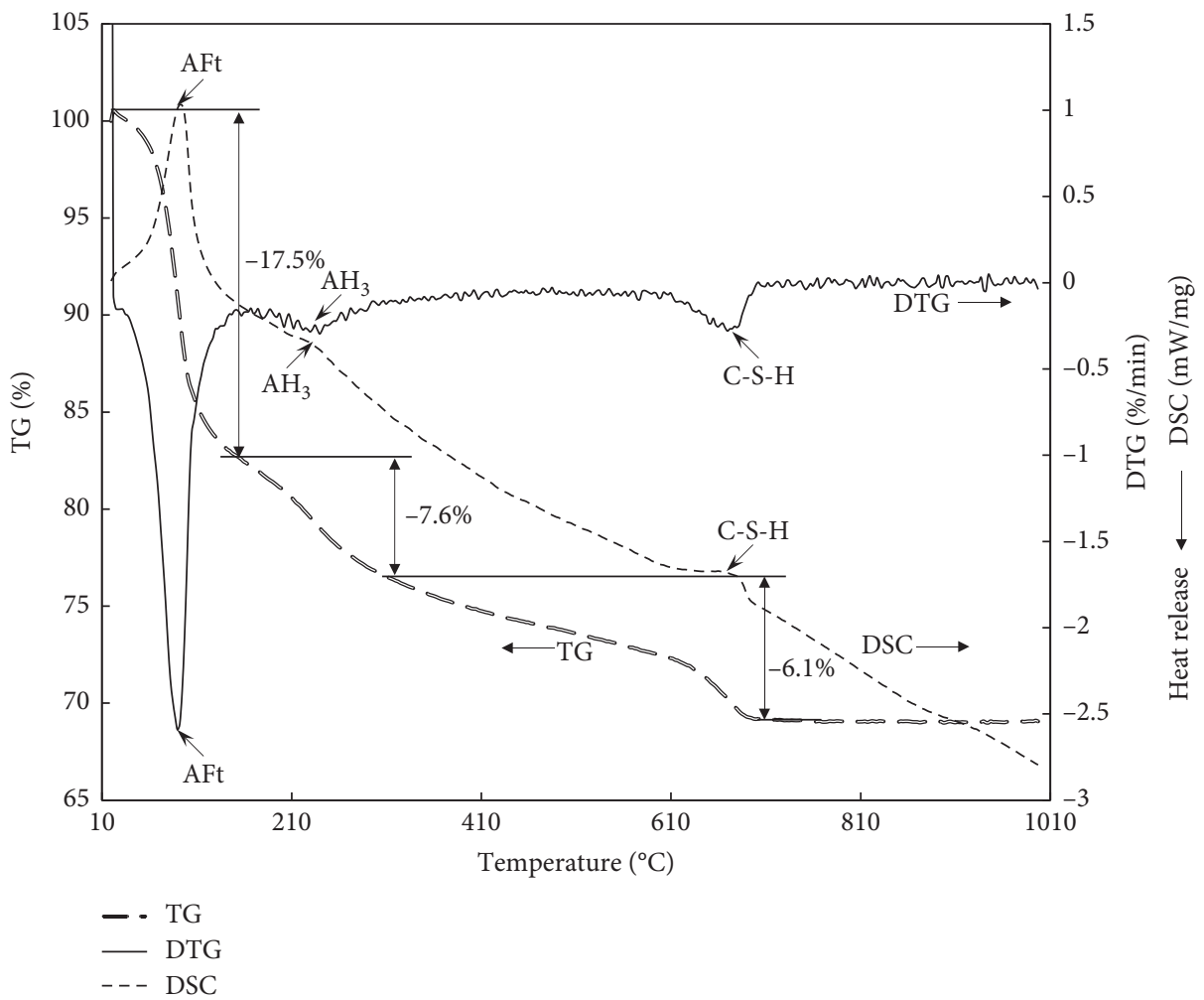

Figure 4: TGA-DSC test curves of NCG concretion.

$$
\begin{aligned}
& W_{i}=W_{L i} \cdot \frac{M_{i}}{n M_{H}}, \\
& W_{i}^{\prime}=\frac{M_{i}}{\sum M_{i}} \times 100,
\end{aligned}
$$

where $W_{i}$ is the mass ratio of solid-phase $i(\%), W_{L i}$ is the mass reduction ratio of solid-phase $i$ at corresponding temperature (\%), $M_{i}$ is the relative molecular weight of solidphase $i, M_{H}$ is the relative molecular weight of water, $n$ is the number of water loss molecules, and $w_{i}^{\prime}$ is the corrected mass ratio of solid-phase $i(\%)$.

Taking the different $\mathrm{W} / \mathrm{C}$ ratio into account, the pore structure characteristics are affected greatly by the concretion, while the proportion of solid-phase production is still relatively stable. The solid composition ratio shown in Figure 5 has a certain degree of representativeness: AFt accounts for the highest proportion, about 65\%; C-S-H occupies the second place, about $20 \%$; and $\mathrm{AH}_{3}$ occupies the least proportion, about $15 \%$.

\section{Nanoscale Pore Characteristic of NCG Concretion}

NCG concretion is a porous structure and the pore size covers the range of nanoscale to micron scale. It is necessary to analyze the pore structure characteristics in different scales by corresponding test and analysis methods. In this 


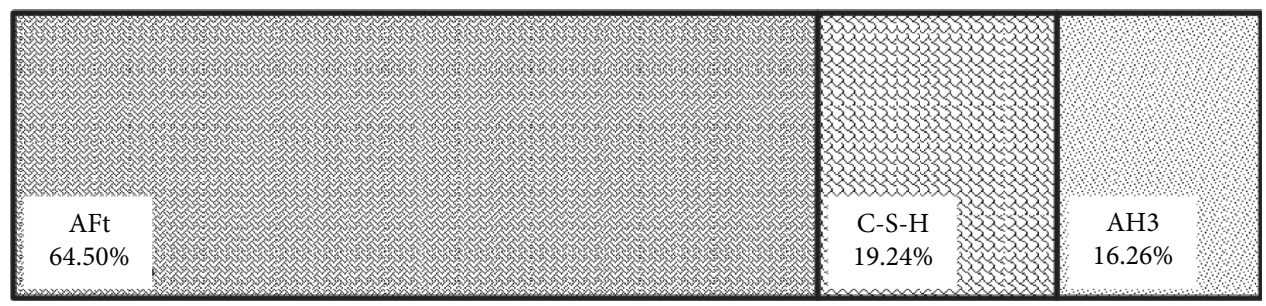

Figure 5: Mass component of solid-phase in of NCG concretion.

paper, BET nitrogen adsorption method and mercury intrusion method (MIP) were used to study the nanoscale pore structure of W2 N NCG concretion.

4.1. Analysis of Pore Characteristic of NCG Concretion by BET. In the BET (Brunauer-Emmett-Teller) test, nitrogen molecules enter into the sample to produce multilayer adsorption under the temperature of liquid nitrogen $(77 \mathrm{~K})$. The 7-day-old W2 N concretion was cut into small pieces, immersed in ethanol (7 days), and dried at constant temperature $\left(60^{\circ} \mathrm{C}\right)$ to stop hydration for the BET test. The adsorption isotherm (curve of the relationship between adsorption medium and pressure when the temperature is constant and the adsorption/desorption equilibrium state is reached) is shown in Figure 6.

According to the classification of adsorption isotherm type and hysteresis loop shape by IUPAC (International Union of Pure and Applied Chemistry) [21], the adsorption isotherm of NCG concretion is type IV and the hysteresis loop is type $\mathrm{H} 3$, without obvious saturated adsorption platform, showing irregular pore structure formed by granular solid-phase and fracture.

Based on the multilayer adsorption model, the relationship equation between the single-layer adsorption capacity $V_{m}$ and the multilayer actual adsorption capacity $V$ is established, which is the BET equation [22] (seen in formula (4)).

$$
\frac{P}{V\left(P_{0}-P\right)}=\frac{C-1}{V_{m} C} \cdot \frac{P}{P_{0}}+\frac{1}{V_{m} C},
$$

where $P$ is the nitrogen partial pressure, $P_{0}$ is nitrogen saturated vapor pressure at liquid nitrogen temperature, $V$ is actual nitrogen adsorption on the sample surface, $V_{m}$ is nitrogen monolayer saturation adsorption capacity, and $C$ is a constant related to the adsorption capacity of a sample.

According to the theory and experience, the BET equation is suitable for $P / P_{0}$ in the range of $0.05-0.3$. The BET diagram $\left(P / V\left(P_{0}-P\right)\right.$ to $\left.P / P_{0}\right)$ obtained from the adsorption isotherm data in the above range (Figure 7 ) is linear, consistent with the expression of BET equation. Comparing the BET equations, the straight slope $A\left(\left(C-1 / V_{m} C\right)\right)$ and intercept $B$ $\left(\left(1 / V_{m} C\right)\right)$ are achieved from BET diagram. Furthermore, the adsorption capacity of monolayer $V_{m}=(1 / A+B)$ can be obtained. Based on the average cross-range area of $\mathrm{N}_{2}$ molecules and Avogadro's constant, the BET specific surface area of NCG concretion is $S_{\mathrm{BET}}=13.418 \mathrm{~m}^{2} / \mathrm{g}$, less than the BET specific surface area of ordinary Portland cement concretion $\left(20.7-58.94 \mathrm{~m}^{2} / \mathrm{g}[23]\right)$.

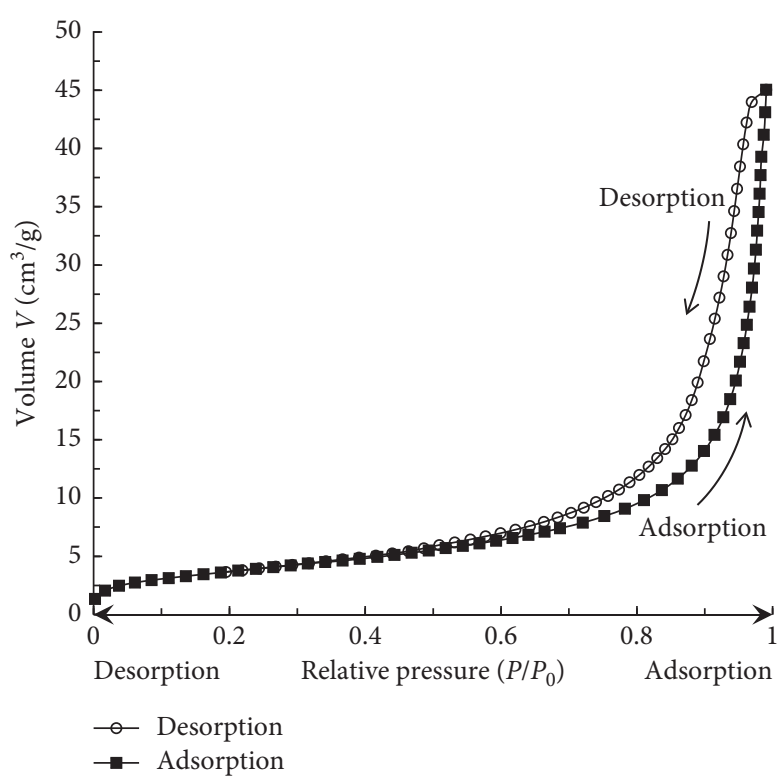

FIgURE 6: The adsorption isotherm of NCG concretion.

Furthermore, according to the adsorption curve data, the pore size distribution diagram of various pore volume related parameters of NCG-concretion is obtained by BJH (Barrett-Joyner-Halenda) method [24], as shown in Figure 8.

In Figure $8, \mathrm{~d} V$ curve represents pore volume per unit aperture and SUM $(\mathrm{d} V)$ represents cumulative pore volume. It can be seen that the pore size of NCG concretion ranges from $2 \mathrm{~nm}$ to $200 \mathrm{~nm}$. The $\mathrm{d} V / \mathrm{d} D$ and $\mathrm{d} V / \mathrm{d} \log D$ differential distribution curves show the variation rate of pore volume with pore diameter. The peak is the most developed pore size, representing the most probable pore diameter (MPD), which is an important characteristic parameter of pore structure. Among them, $\mathrm{d} V / \mathrm{d} \log D$ curve is more intuitive when the pore size distribution is large. According to Figure 8 , the $\mathrm{d} V / \mathrm{d} \log D$ curve has a significant peak at $113.57 \mathrm{~nm}$, indicating that the pore size is the most probable diameter. According to IUPAC classification criteria based on pore size (micropore diameter $\leq 2 \mathrm{~nm}$, mesopore diameter between $2 \mathrm{~nm}$ and $50 \mathrm{~nm}$, and macropore diameter $\geq 50 \mathrm{~nm}$ ), the pores of NCG concretion belong to the mesopores and macropores by BET test. In order to analyze the pore size distribution more scientifically and reasonably, the range of mesopore and macropore is further divided into four ranges: $2 \mathrm{~nm}-10 \mathrm{~nm}, 10 \mathrm{~nm}-50 \mathrm{~nm}, 50 \mathrm{~nm}-100 \mathrm{~nm}$, and $100 \mathrm{~nm}-200 \mathrm{~nm}$. According to the distribution of pore 


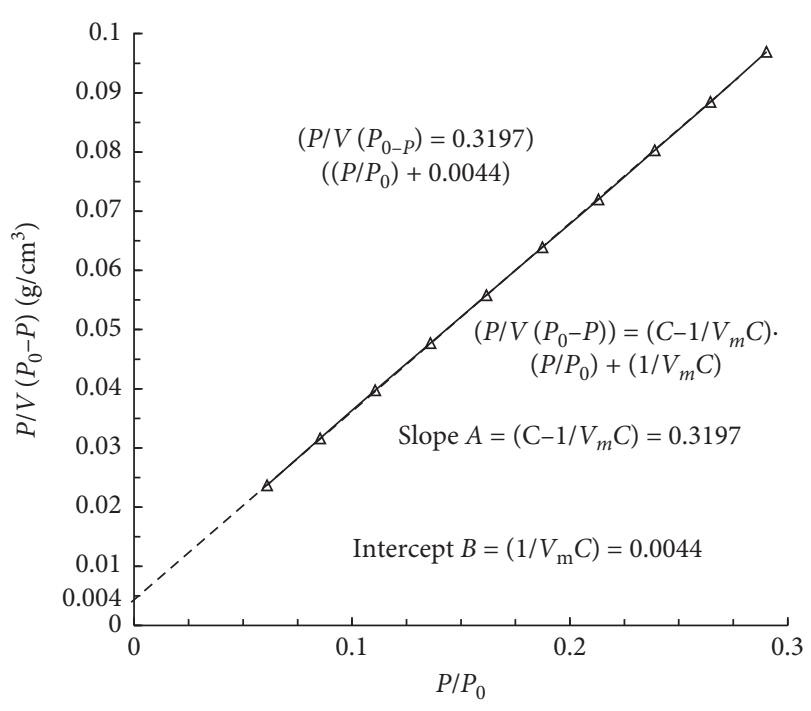

Figure 7: The BET figure of NCG concretion.

volume $(\mathrm{d} V)$ of BET test, the proportion of pore volume of four ranges can be obtained, as shown in Figure 9.

4.2. Analysis of Pore Characteristic of NCG Concretion by MIP. According to the results of BET test, the pore size distribution of NCG concretion concentrates in the range of mesopore and macropore, with the most probable pore diameter belonging to macropore. In order to obtain more accurate pore size distribution characteristics in macropore size range, the pore structure is further tested by mercury intrusion porosimetry (MIP). The amount of mercury entering the pore under different external pressure is measured, and the pore diameter is estimated based on the Washburn equation [25]:

$$
r=\frac{4 \sigma \cos \theta}{P}
$$

where $r$ is pore diameter, $p$ is the pressure measuring pore, $\sigma$ is the surface tension of mercury $(0.485 \mathrm{~N} / \mathrm{m})$, and $\theta$ is the contact angle between mercury and material $\left(130^{\circ}\right)$.

In this paper, the 7-day-old $\mathrm{W} 1 \mathrm{~N}$ and $\mathrm{W} 2 \mathrm{~N}$ concretions and the 28-day-old $\mathrm{W} 1 \mathrm{~N}$ concretion were cut into small pieces, immersed in absolute ethanol (7 days), and dried at constant temperature $\left(60^{\circ} \mathrm{C}\right)$ to stop the hydration reaction for MIP test, obtaining the mercury intrusion and extrusion curves shown in Figure 10.

According to the range of mercury pressure value $P$ in the mercury intrusion and extrusion curves in Figure 10, it can be concluded from equation (5) that the pore size distribution of NCG concretion is between $3.2 \mathrm{~nm}$ and $4.2 \times 10^{5} \mathrm{~nm}$, with wide pore size coverage. According to the MIP test data, the log differential curve of pore volume to pore diameter $(\mathrm{dV} / \mathrm{d}$ $\log \mathrm{D}$ ) in logarithmic coordinates and the percentage of pore volume to total pore volume $(\mathrm{dV} / \mathrm{V})$ under different pore sizes were obtained, as shown in Figures 11-13.

According to IUPAC classification criteria based on pore size, the pores of NCG concretions are mesopores and macropores, far exceeding the limit of macropores $\geq 50 \mathrm{~nm}$ defined before. In order to analyze the whole range aperture distribution scientifically and reasonably, this paper subdivides the macropore $(\geq 50 \mathrm{~nm})$, considering that the MPD $(\mathrm{d} V / \mathrm{d} \log D$ peak pore diameter) is roughly located in the middle of each pore size range. The subdivided results are shown in Table 2.

Focusing on the above pore size ranges, the $\mathrm{d} V / \mathrm{d} \log D$ values and the coefficient of variation (C. V.) of the MPD (Figure 14) and the percentage and its C. V. of pore volume accumulated (Figure 15) of W1 N and W2 N concretions with 7 days of age are compared. In addition, the $\mathrm{d} V / \mathrm{d} \log D$ values and the C. V. of the MPD (Figure 16) and the percentage and its C. V. of pore volume accumulated (Figure 17) of W1 N concretion with 7 days of age and 28 days of age are compared.

According to the above analysis chart, the conclusion of nanoscale pore-phase distribution of NCG concretion can be drawn. (1) The pore size of NCG concretion mainly concentrates in the range of $50 \mathrm{~nm}$ to $3000 \mathrm{~nm}$, and the pore volume accounts for about $90 \%$ of the total pore volume (referred to as the rich pore range). Moreover, the MPD also appears in the range of $700 \mathrm{~nm}-900 \mathrm{~nm}$. The pore size distribution of the rich pore range is representative and can be used as the pore-phase characteristic parameter of NCG concretion. (2) The occurrence probability in each pore size range and the proportion of pore volume in the rich pore range are significantly higher than those in other pore size ranges, and the dispersion degree of pore diameter occurrence probability and volume proportion is also higher than that of other ranges, but it is lower than that of the whole pore range. (3) With the increase of $\mathrm{W} / \mathrm{C}$ ratio and curing age, the MPD decreases, while the probability of the MPD increases, and the dispersion of the occurrence probability in each pore size range increases. (4) The proportion of pore volume and the dispersion of pore volume proportion increase as the $\mathrm{W} / \mathrm{C}$ ratio and curing age increase.

In the analysis of MIP test, according to a series of parameters, such as the volume and mass of the dilatometer module of the mercury injection meter, the mercury mass before and after pressure, the mercury density and the test sample mass, and the macroscopic physical parameters, porosity $\eta$, bulk density $\rho_{B}$, and skeletal density $\rho_{S}$, can be obtained, shown in Table 3. It can be seen that the porosity of NCG concretion is more than $60 \%$, belonging to a kind of porous material. With the increase of W/C ratio and curing age, the porosity of concretion increases and the bulk density decreases. The skeletal density increased slightly as the W/C ratio increased, while the skeletal density decreased slightly with the increase of age. In contrast, the $\mathrm{W} / \mathrm{C}$ ratio and curing age have little effect on the solid density of the concretion, mainly on the porosity, resulting in the change of the bulk density.

\section{Microstructure of NCG Concretion}

5.1. 3D Reconstruction on Microstructure of NCG Concretion. In this paper, 3D X-ray Microscopy (3D-XRM) technology was used to analyze the NCG concretion to obtain the multiphase 3D microscopic morphology of the concretion.

In this paper, 3D-XRM projection images of 7-day-old W1 N and W4 N concretions were collected, and 1004 2D images of $\mathrm{W} 1 \mathrm{~N}$ concretion samples (resolution $5.15 \mu \mathrm{m}$ ) 


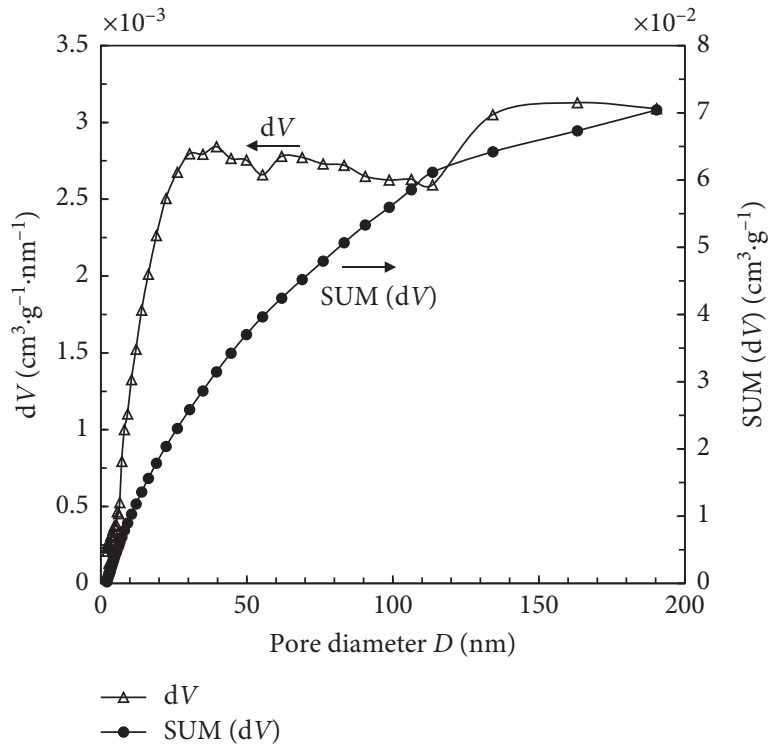

(a)

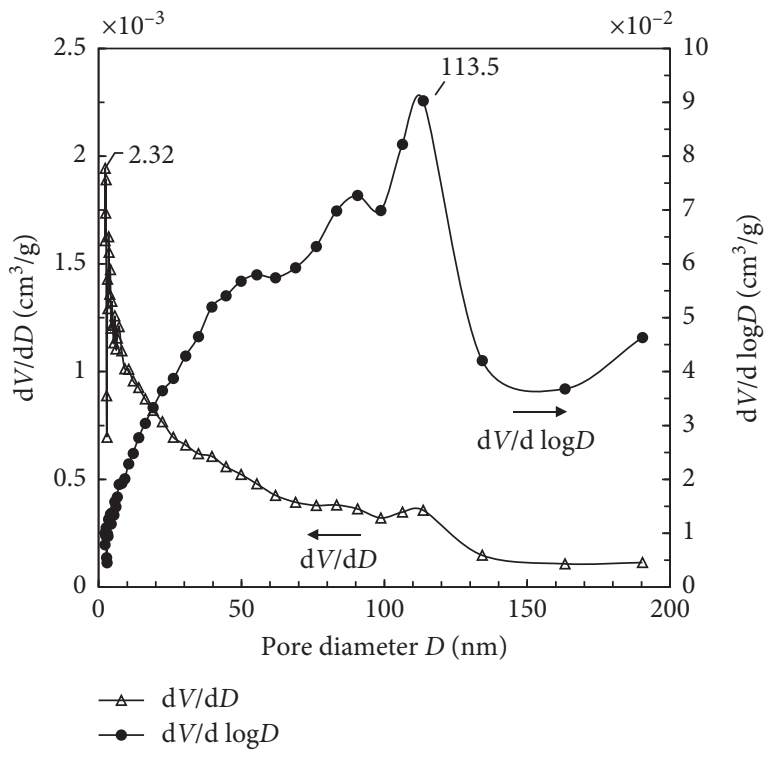

(b)

Figure 8: The pore-diameter distribution of NCG concretion by BET test. (a) $\mathrm{d} V$ and SUM (d $V$ ). (b) $\mathrm{d} V / \mathrm{d} D$ and $\mathrm{d} V / \mathrm{d} \log D$.

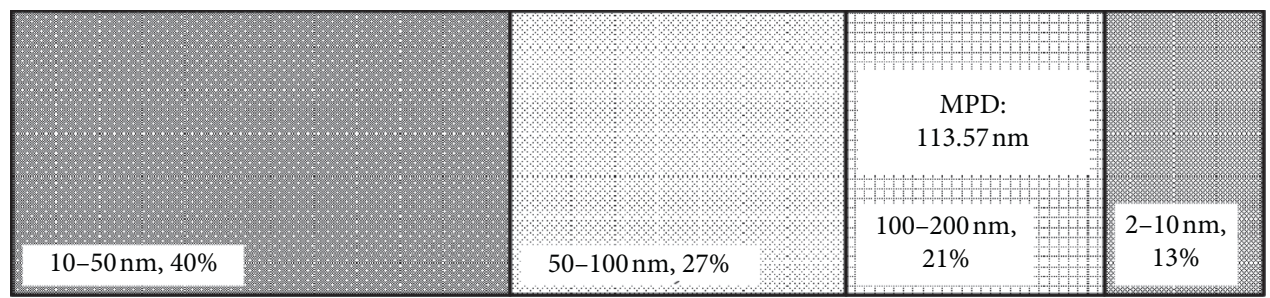

FIGURE 9: Pore volume proportion of NCG concretion in various pore-diameter ranges by BET test.

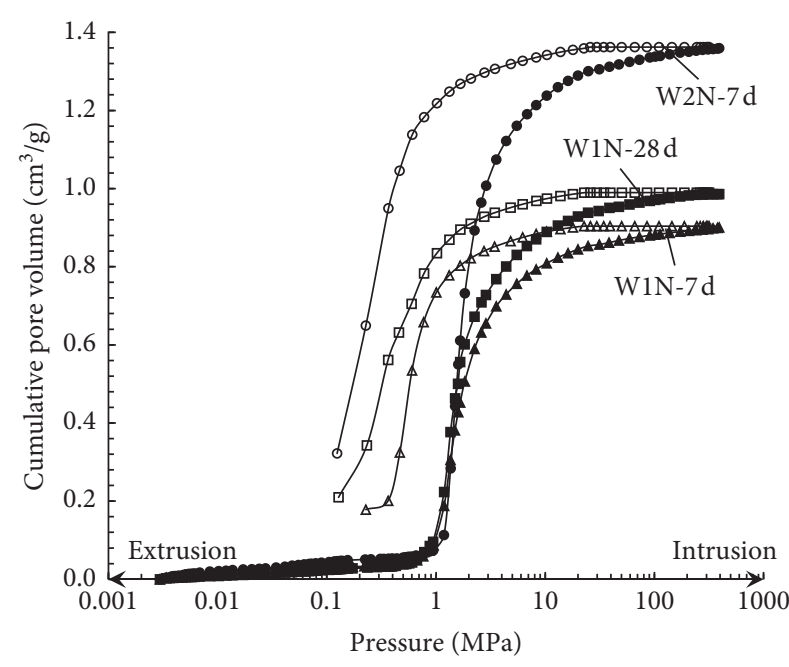

$\rightarrow$ W1N-7d-intrusion $\leftrightarrow$ W1N-7d-extrusion
$\rightarrow$ W2N-7d-intrusion $\leftrightarrow$ W2N-7 d-extrusion
$\rightarrow$ W1N-28d-intrusion

Figure 10: Mercury intrusion and extrusion curves of NCG concretion. and $10172 \mathrm{D}$ images of W4 $\mathrm{N}$ concretion samples (resolution $5.45 \mu \mathrm{m})$ were obtained. Combined with the composition of NCG concretion, the segmentation and extraction of porephase, AFt-phase, and gel-phase (several gels cannot be differentiated, so they are combined into one phase for statistical analysis) can be realized by means of 2D image gray threshold. Taking $\mathrm{W} 1 \mathrm{~N}$ as an example, the distribution of each phase at the same position is shown in Figure 18.

$3 \mathrm{D}$ reconstruction of more than $10002 \mathrm{D}$ images of two types of NCG concretions was carried out, including threephase holistic and hidden solid-phases (AFt-phase and gelphase) showing only the three-dimensional reconstruction state of pores. Taking $\mathrm{W} 4 \mathrm{~N}$ as an example, the $3 \mathrm{D}$ reconstruction is shown in Figure 19.

At a resolution of about $5 \mu \mathrm{m}$, the micron scale of the images acquired by 3D-XRM can be recognized at about $10 \mu \mathrm{m}$. As can be seen from the above $2 \mathrm{D}$ images and $3 \mathrm{D}$ reconstructed images, AFt accounts for the largest proportion in the NCG concretion at this micron scale and plays the role of the main skeleton. There were obvious differences between $\mathrm{W} 1 \mathrm{~N}$ and $\mathrm{W} 4 \mathrm{~N}$ concretions. In $\mathrm{W} 1 \mathrm{~N}$ concretion, AFt-phase was more uniform and dense, gel-phase accounted for a larger proportion, and pore-phase also 


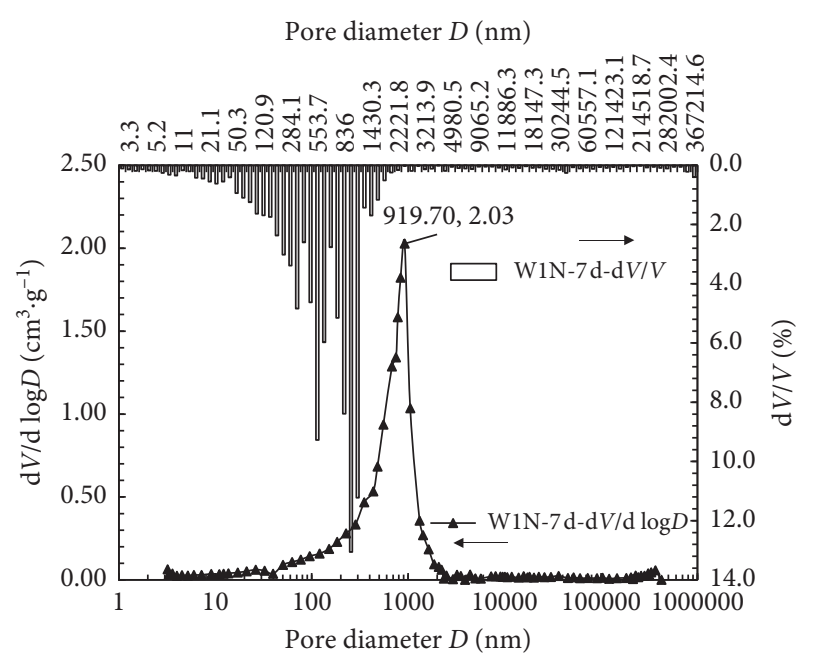

FIGURe 11: Pore-diameter distribution of 7-day-old W1 N concretion by MIP test.

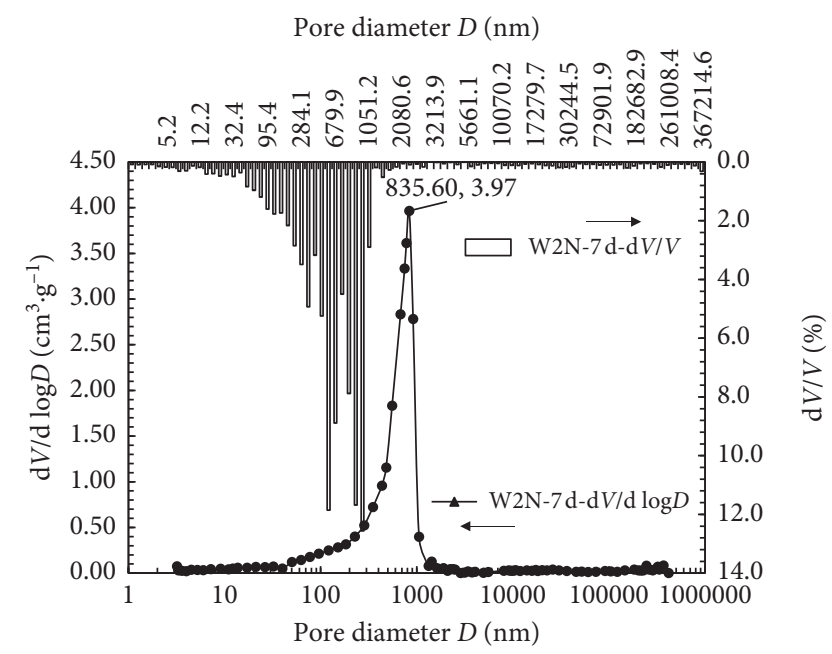

FIgure 12: Pore diameter distribution of 7-day-old W2 $\mathrm{N}$ concretion by MIP test.

accounted for a smaller proportion. Further quantitative statistics of the volume ratio of each phase of the two types of specimens are shown in Figure 20.

It can be seen that when the $\mathrm{W} / \mathrm{C}$ ratio increases and foaming agent is added, the ratio of AFt-phase in the concretion is basically stable, but the ratio of the gel-phase is reduced by $68.15 \%$, and the ratio of the pore-phase is increased by $162.37 \%$.

For W1N concretion, according to 3D-XRM analysis, the pore-phase proportion is $3.80 \%$, and the porosity obtained by MIP analysis (Chapter 4.2 ) is $67.71 \%$. The differences of the above data results are analyzed. The difference of data is mainly due to the different interval of statistical aperture identified by the two test analysis methods; the pore size range of $3 \mathrm{D}-\mathrm{XRM}$ analysis is more than $10 \mu \mathrm{m}$. Nanoscale pores, especially the smaller pores in $\mathrm{AFt}$, cannot be identified and counted. However, the pore size range of MIP test statistics is between $2 \mathrm{~nm}$ and $400 \mu \mathrm{m}$,

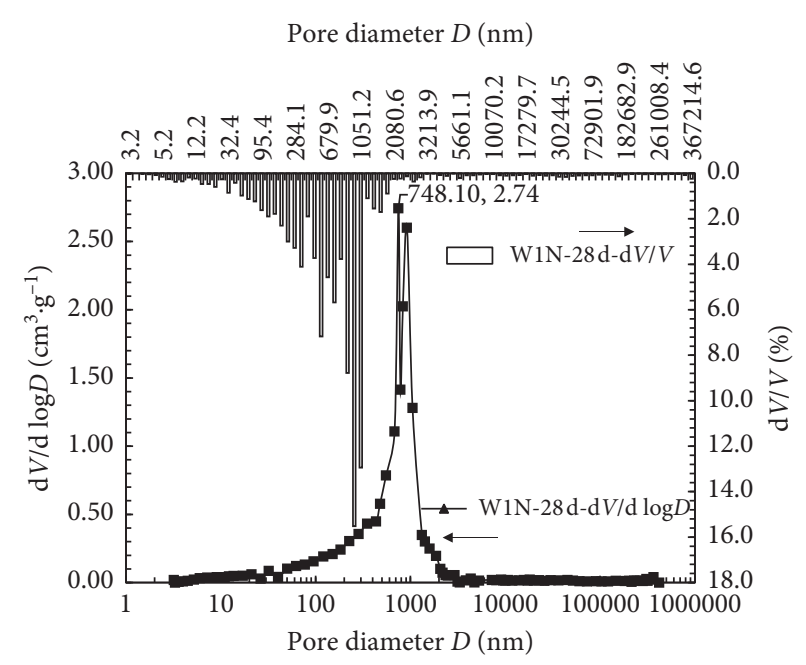

FIGURE 13: Pore diameter distribution of 28-day-old W1 N concretion by MIP test.

so the pore size range of MIP statistics is wider, and the porosity is higher. The overall porosity of MIP is $67.71 \%$, among which the proportion of pore volume over $10 \mu \mathrm{m}$ in the total pore diameter range is $3.24 \%$, and the proportion of pore-phase over $10 \mu \mathrm{m}$ after conversion is $2.14 \%$, which is basically consistent with the results of 3D-XRM analysis. The two test analysis results are mutually verified.

Further, according to the 3D-XRM test data, quantitative statistics were made on the volume, surface area, quantity, distribution, and other information of the monomer of the pore-phase and gel-phase, respectively (because the proportion of AFt-phase is too high to distinguish the monomers, the statistics are not made). In order to further facilitate comparative analysis, the equivalent diameter of an equal volume sphere $\mathrm{d}_{V}$ (diameter of a sphere of the same volume: $d_{V}=\sqrt[3]{6 V / \pi}$. The equivalent diameter mentioned in the following paper refers to that of an equal volume sphere) corresponding to monomer volume $V$ of each pore-phase is counted. In this paper, the monomer quantity, volume interval, and equivalent-diameter interval of each phase are counted by the two types of samples, as shown in Table 4.

In order to facilitate the data analysis and processing, the equivalent-diameter of the equal volume sphere is divided into several intervals with the accuracy of $1 \mu \mathrm{m}$. The relative parameters of pore-phase or gel-phase monomer were counted, including: (1) the quantity $n$; (2) the representative value of equivalent-diameter $d_{i}$ (the representative value refers to the average value of relevant parameters of all monomers in the $1 \mu \mathrm{m}$ interval; the same below); (3) interval volume $V i$ (refers to the sum of all monomer volumes in $1 \mu \mathrm{m}$ interval corresponding to the representative value of equivalent-diameter $d_{i}$ ); (4) surface area representative value $S_{i}$; (5) the surface area $S_{i E D}$ calculated according to the representative value of equivalent-diameter; (6) according to $S_{i}$ and $S_{i E D}$, the representative value of sphericity $\phi_{s}$ of the interval monomer is obtained $\left(\phi_{s}=S_{i} / S_{i E D}\right.$, parameters for characterizing the morphology of the monomer, the closer 
TABLe 2: Pore types boundary based on pore diameter $D$.

\begin{tabular}{lccccc}
\hline \multirow{2}{*}{ Pore diameter range/(nm) } & $0<D \leq 2$ & $2<D \leq 50$ & \multicolumn{2}{c}{$D \geq 50$ (IUPAC macropore, subdivided) } \\
& (Same as IUPAC) & (Same as IUPAC) & $50<D \leq 3000$ & $3000<D \leq 50000$ & $D>50000$ \\
\hline Category & Micropore & Mesopore & Secondary macropore & Middle macropore & Extra macropore \\
\hline
\end{tabular}

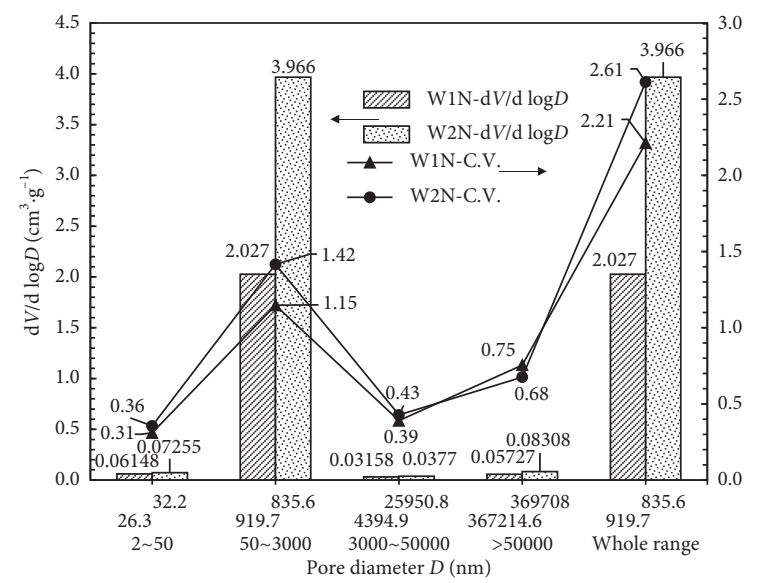

Figure 14: The $\mathrm{d} V / \mathrm{d} \log D$ of MPD and its C. V. in pore-diameter ranges of different $\mathrm{W} / \mathrm{C}$ ratio NCG concretion.

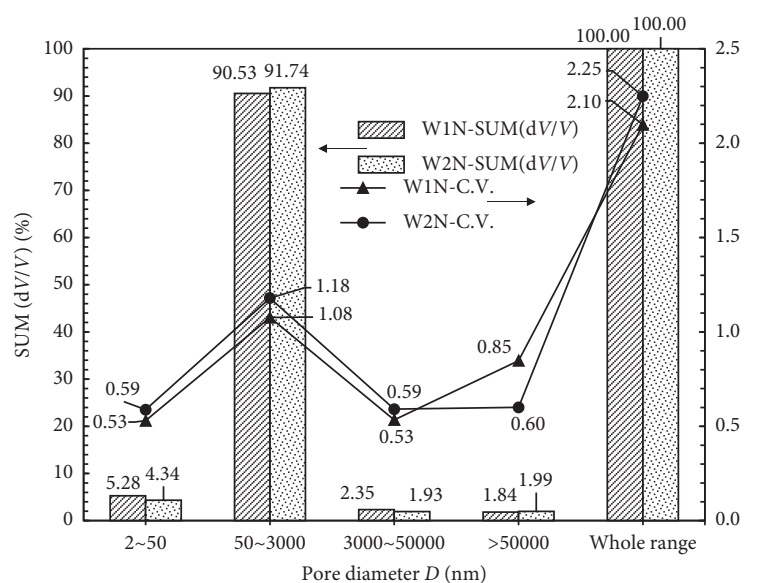

FIgURE 15: The cumulative pore volume proportion and its C. V. in pore-diameter ranges of different W/C ratio NCG concretion.

to 1 , the closer the monomer to the sphere). In addition, in order to compare and analyze the volume distribution of monomer in each phase of the two types of concretions, in view of the differences in the total volume of the two types of concretions, the direct comparison of the interval volume cannot truly reflect the differences between the two types of concretions, so the interval volume proportion $V_{i}$ (the percentage of the interval volume $V_{i}$ in the total volume of all monomers) is introduced.

5.2. Micron-Scale Analysis of Pore-Phase of NCG Concretion. In order to analyze the regularity of the continuity of the equivalent-diameter of the pore-phase in each area, the equivalent-diameter is numbered from the small to the large, forming the pore-label. According to the 3D-XRM analysis

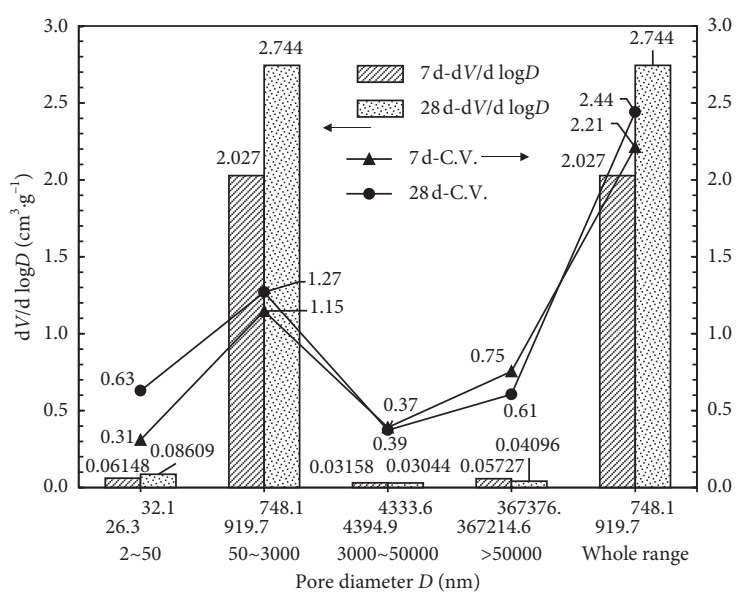

Figure 16: The $\mathrm{d} V / \mathrm{d} \log D$ of MPD and its C. V. in pore-diameter ranges of different-ages NCG concretion.

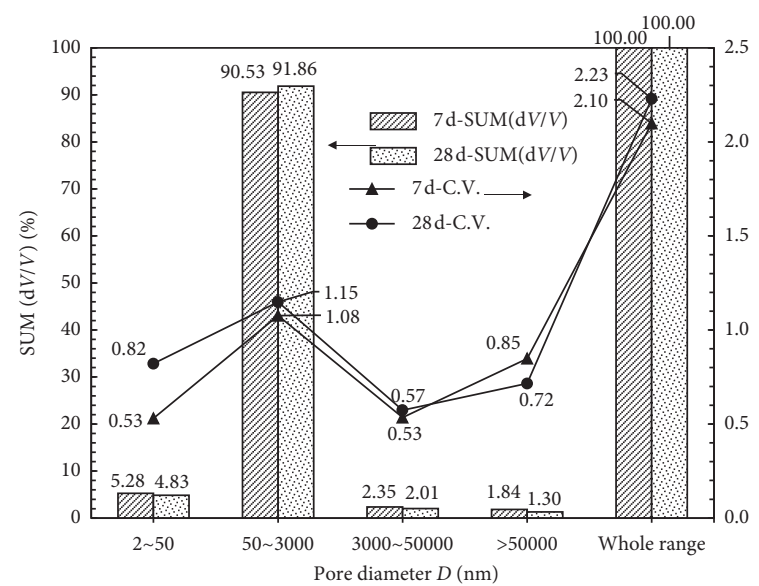

FIgURE 17: The cumulative pore volume proportion and its C. V. in pore-diameter ranges of different-ages NCG concretion.

data, the curves of the relationship between the equivalentdiameter of the two types of concretions and the pore-label sequence and the number of monomers are obtained, as shown in Figure 21.

The pore-phase distribution curves of $\mathrm{W} 1 \mathrm{~N}$ and $\mathrm{W} 4 \mathrm{~N}$ are basically consistent. The pore-label curve in Figure 21 shows the continuity characteristics of pore-phase equivalent-diameter distribution from small to large. The front range is basically straight line, indicating that the pore of the small equivalent-diameter increases uniformly and continuously at a similar amplitude. When the pore equivalentdiameter reaches $169.0 \mu \mathrm{m}$, the slope of the curve becomes slower, indicating that the growth of diameter is no longer uniform and there is a leaping growth. The bigger the diameter, the bigger the leaping growth rate. The quantity 
TABLe 3: Porosity and density of NCG concretion. The data of "the rate of change" is the rate of change relative to W1 N (7 d).

\begin{tabular}{lcccccc}
\hline \multirow{2}{*}{ Samples (age) } & \multicolumn{2}{c}{ Porosity $\eta /(\%)$} & \multicolumn{2}{c}{ Bulk density $\rho_{B} /\left(\mathrm{g} / \mathrm{cm}^{3}\right)$} & \multicolumn{2}{c}{ Skeletal density $\rho_{S} /\left(\mathrm{g} / \mathrm{cm}^{3}\right)$} \\
& Value & Rate of change & Value & Rate of change & Value & Rate of change \\
\hline W1 N (7 d) & 65.91 & -- & 0.7319 & -- & 2.1472 \\
W2 N (7 d) & 74.77 & $13.44 \%$ & 0.5500 & $-24.85 \%$ & 2.1801 & $1.53 \%$ \\
W1 N (28d) & 67.71 & $2.73 \%$ & 0.6867 & $-6.18 \%$ & 2.1271 & $-0.94 \%$ \\
\hline
\end{tabular}

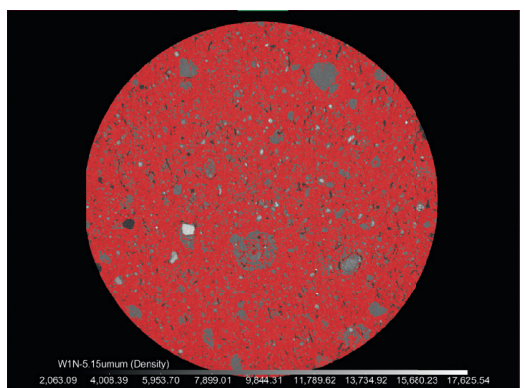

(a)

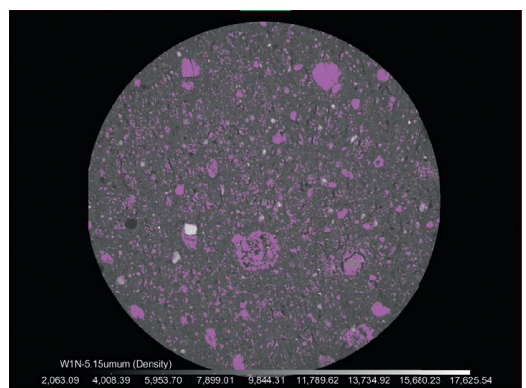

(b)

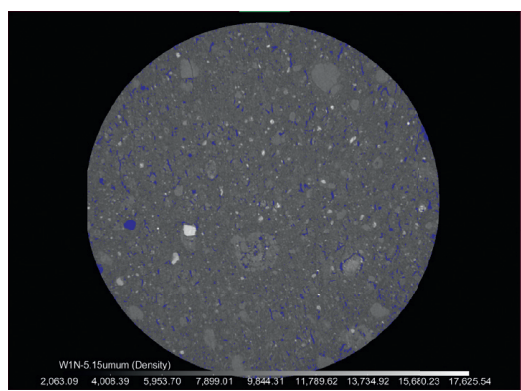

(c)

FIgURE 18: Extraction of AFt-phase of NCG concretion by 3D-XRM. (a) AFt-phase. (b) Gel-phase. (c) Pore-phase.

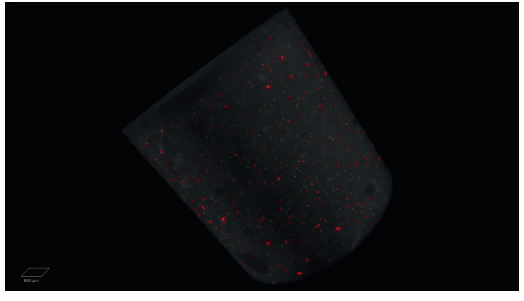

(a)

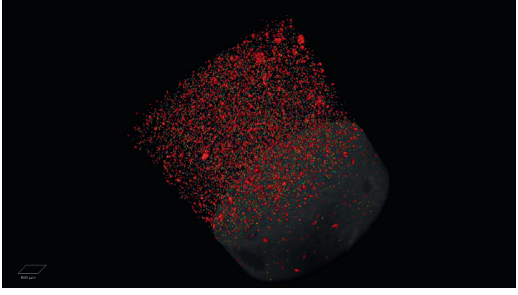

(b)

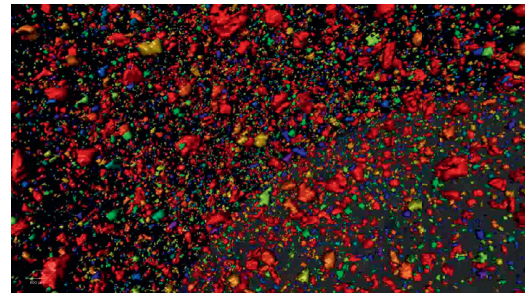

(c)

FIGURE 19: 3D reconstruction of NCG concretion by 3D-XRM. (a) Whole three-phase. (b) Pore-phase. (c) Pore-phase magnification.

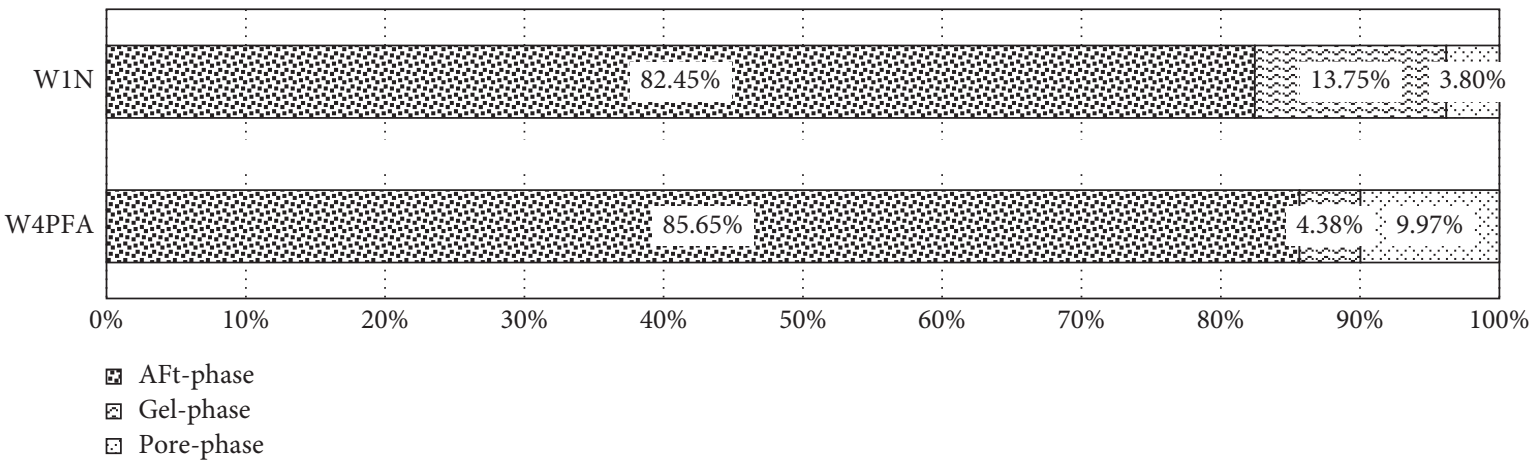

FIGURE 20: Volume proportion of phases of NCG concretion by 3D-XRM.

curve in Figure 21 shows that the pore-phase quantity distribution basically follows the rule that the larger the diameter is, the smaller the quantity is. According to the characteristics of the quantity distribution curve, it can be roughly divided into three ranges: the small aperture range with steep descent, the medium aperture range with curved descent, and the large aperture range with basically horizontal appearance. Among them, the feature points of the front range were taken from the point where the slope of steep range and curved range changed (around $40.5 \mu \mathrm{m}$ ), while the feature points of the back range were taken from the aperture continuity boundary of the pore-label curve (around $169.0 \mu \mathrm{m}$ ), as shown in Figure 21. The characteristics of the three ranges of the equivalent-diameter quantity curve are as follows: (1) small aperture range (23.4/ $26.5 \mu \mathrm{m}-40.5 \mu \mathrm{m})$ : the number is large, and the number decreases sharply with the increase of equivalent diameter; (2) medium aperture range $(40.5 \mu \mathrm{m}-169.0 \mu \mathrm{m})$ : the number 
TABLE 4: Numbers, volume range, and equivalent-diameter range of monomers by 3D-XRM.

\begin{tabular}{|c|c|c|c|}
\hline \multicolumn{2}{|c|}{ Statistical parameters of 3D-XRM } & W1 N & W4 N \\
\hline Pore-phase & $\begin{array}{l}\text { Number of monomers } \\
\text { Volume interval } / \mu \mathrm{m}^{3} \\
\text { Equivalent diameter } / \mu \mathrm{m}\end{array}$ & $\begin{array}{c}54078 \\
6.01 \times 10^{3}-1.32 \times 10^{8} \\
23.45-632.50\end{array}$ & $\begin{array}{c}46452 \\
8.97 \times 10^{3}-64.71 \times 10^{8} \\
26.51-638.98\end{array}$ \\
\hline Gel-phase & $\begin{array}{l}\text { Number of monomers } \\
\text { Volume interval } / \mu \mathrm{m}^{3} \\
\text { Equivalent diameter } / \mu \mathrm{m}\end{array}$ & $\begin{array}{c}111500 \\
1.04 \times 10^{3}-8.21 \times 10^{7} \\
27.07-539.25\end{array}$ & $\begin{array}{c}189618 \\
2.24 \times 10^{3}-3.21 \times 10^{7} \\
16.24-394.27\end{array}$ \\
\hline
\end{tabular}

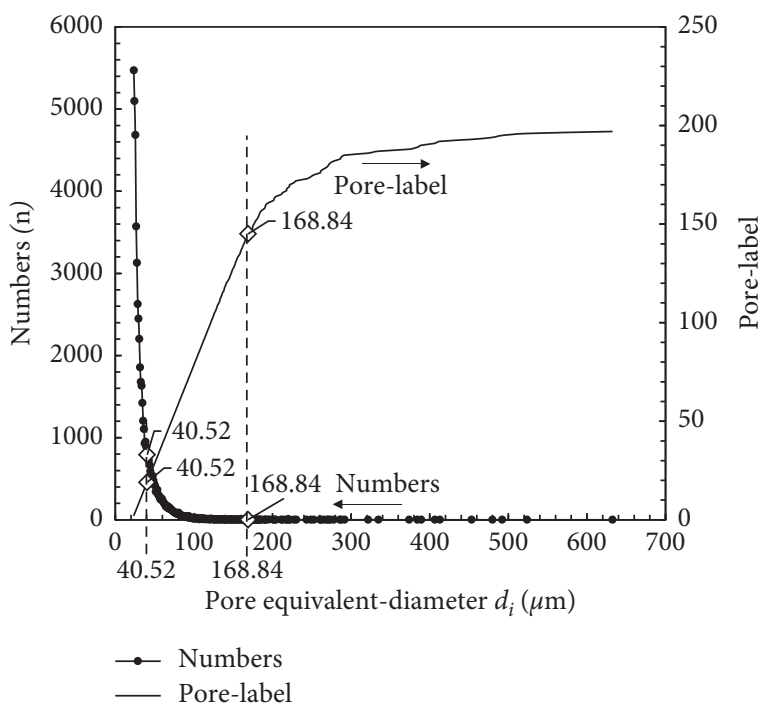

(a)

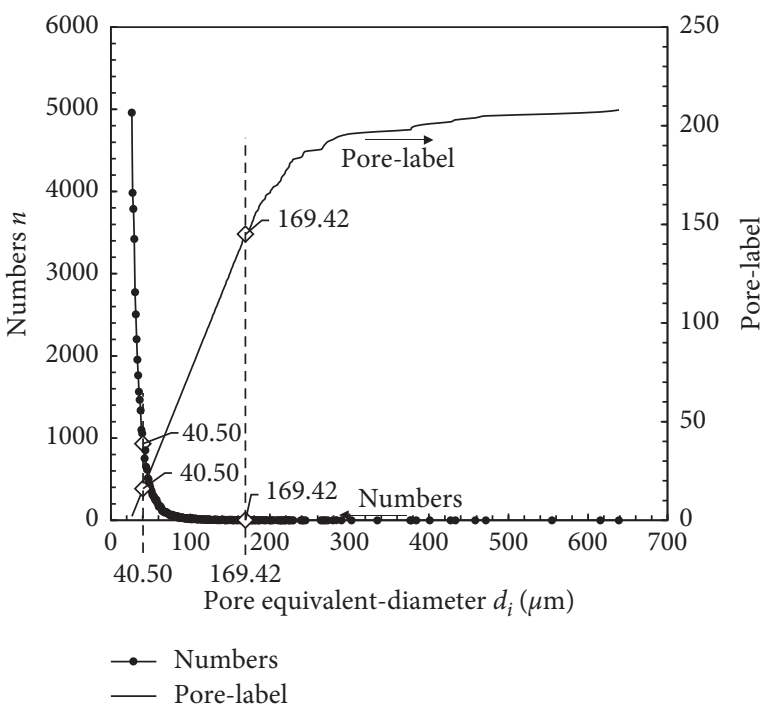

(b)

FIgURe 21: Quantity and continuity distribution of pore-phase of NCG concretion by 3D-XRM. (a) W1 N. (b) W4 N.

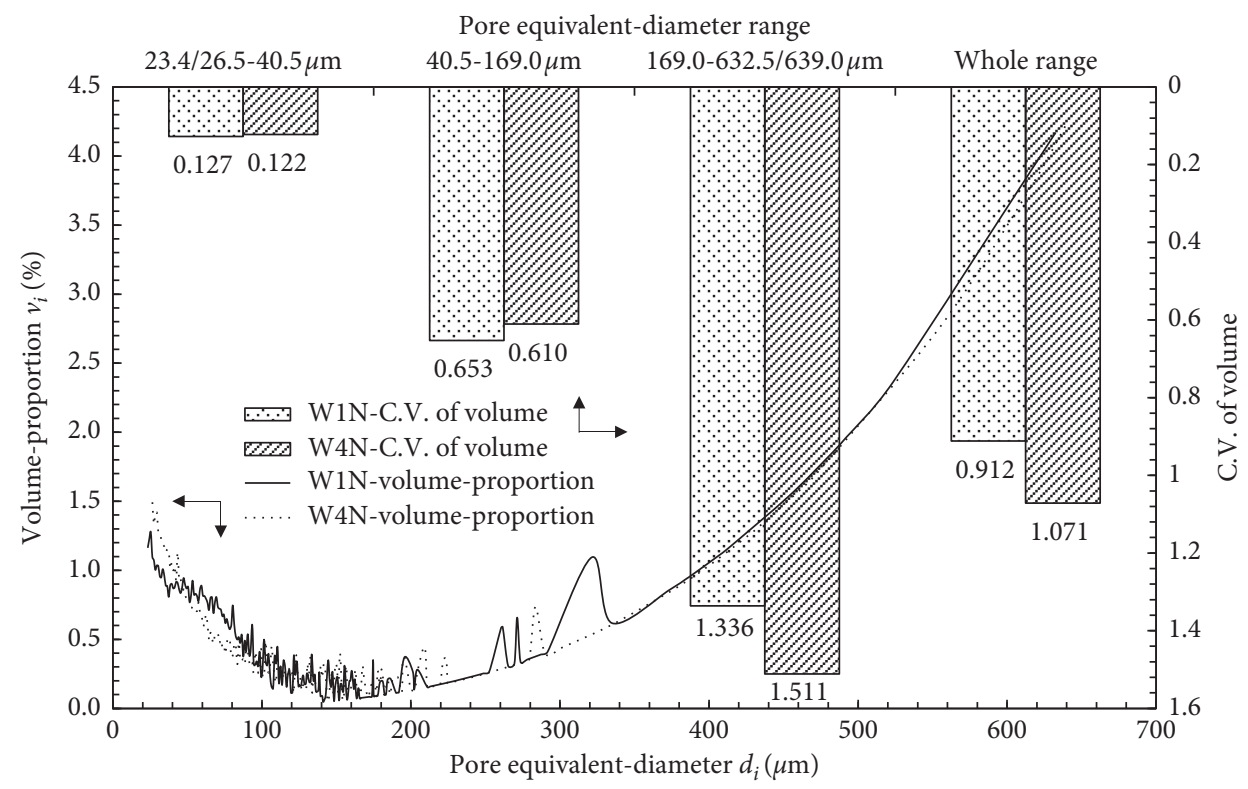

FIGURE 22: Volume-proportion distribution and C. V. of volume of pore-phase of NCG concretion by 3D-XRM.

is in the middle, and the slope of the number decreases with the decrease of the equivalent-diameter; (3) large aperture range $(169.0 \mu \mathrm{m}-632.5 / 639.0 \mu \mathrm{m})$ : the number is small, the number is basically maintained at single digit, basically stable, and does not change with the equivalent-diameter, and the quantity and diameter have great randomness. 


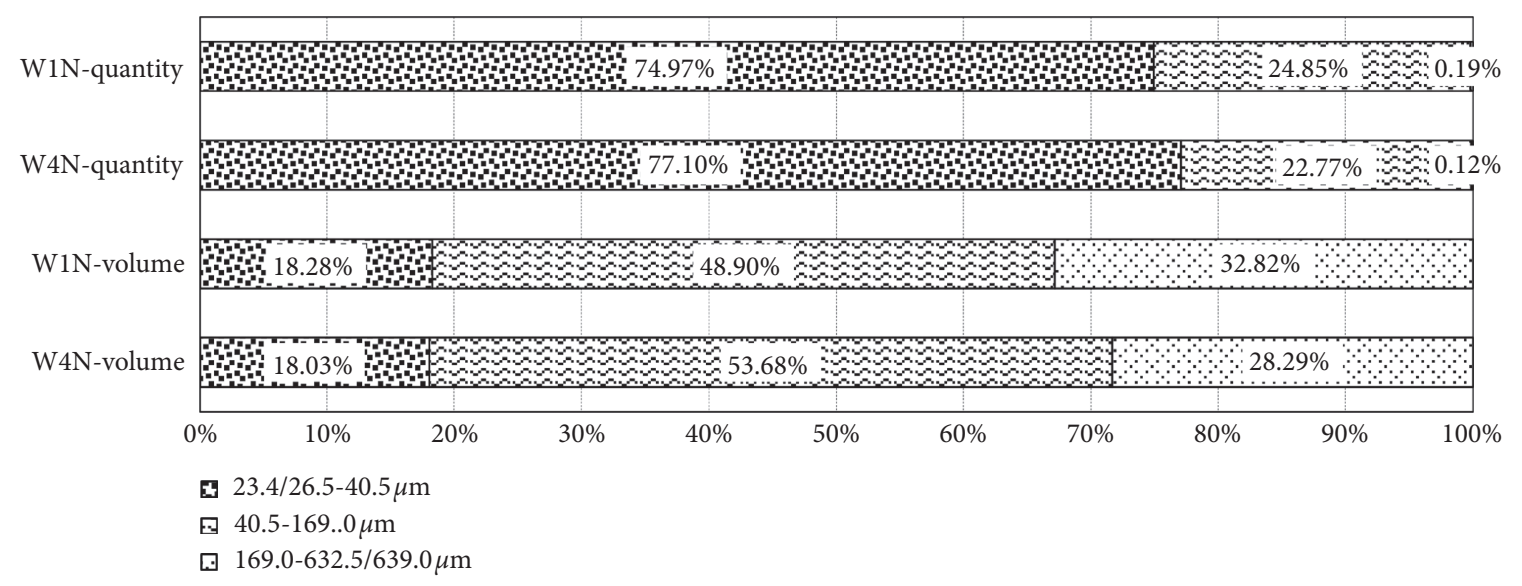

FIGURE 23: Quantity and volume proportion in different pore equivalent-diameter ranges of NCG concretion by 3D-XRM.

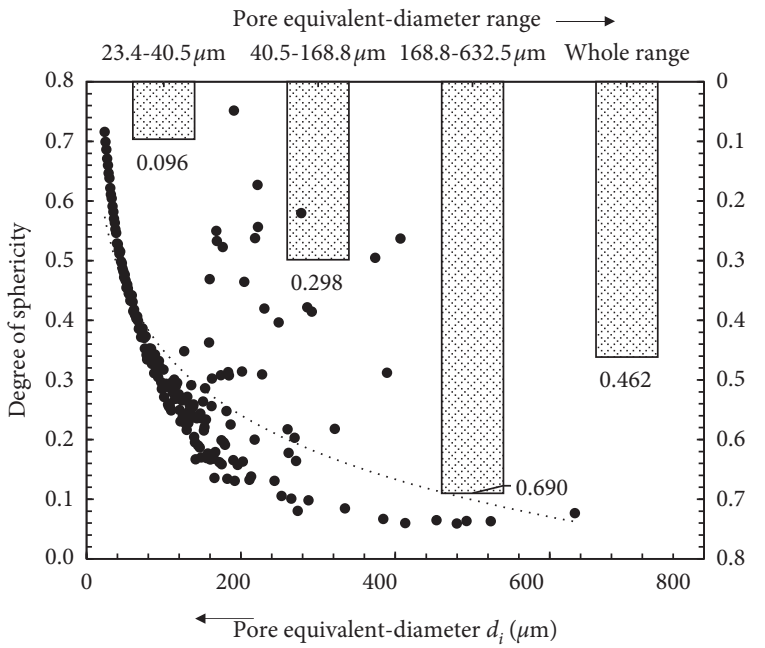

(a)

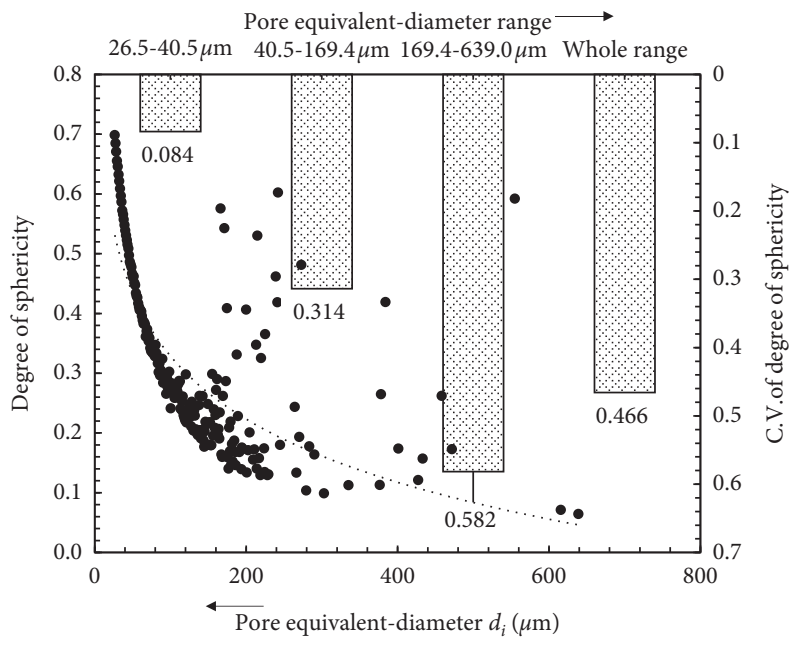

(b)

FIgURe 24: Sphericity distribution and its C. V. of pore-phase of NCG concretion by 3D-XRM. (a) W1 N. (b) W4 N.

Further statistics of the interval volume proportion distribution corresponding to the equivalent diameter of the two types of concretion and the coefficient of variation (C. V.) of pore-phase monomer volume in the three diameter ranges are shown in Figure 22.

The volume distribution curve in Figure 22 refers to the total volume of all pore-phase monomers corresponding to the representative value of equivalent-diameter. It can be seen that the volume distribution curves of W1 N and W4 N show a similar law of first decreasing and then rising: when the pore diameter is small, although the volume of single pore-phase monomer is small but the number is large, the corresponding total volume is large. With the increase of pore diameter, although the volume of pore-phase monomer increases, the number decreases correspondingly, and the corresponding total volume shows a decreasing trend. Then, as the decreasing trend of the quantity of pore-phase monomers slowed down its volume rendering again with the trend of the equivalent diameter increases. Comparing the volume distribution curves of $\mathrm{W} 1 \mathrm{~N}$ and $\mathrm{W} 4 \mathrm{~N}$, it can be seen that the volume distribution curves of the two types of concretion fluctuate significantly in the small aperture range, and there are obvious differences between the two types of curves. This is mainly due to the large difference in the number of monomers in the corresponding $1 \mu \mathrm{m}$ unit interval when the equivalent-diameter is small. However, when the equivalent-diameter is large, the two types of concretion are stable in the corresponding unit interval of monomer number region, so the two types of curves tend to be consistent. Generally speaking, the pore-phase volume distribution of the two types of concretion is relatively close. It can be seen that the $\mathrm{C}$. V. of volume of each monomer in the small diameter range is the smallest, which means that the volume of the pore-phase monomer tends to be consistent, while the dispersion coefficient in the medium diameter range and the large diameter range increases gradually, indicating that the volume difference of the porephase monomer increases with the increase of the equivalent diameter. The C. V. of volume of $\mathrm{W} 1 \mathrm{~N}$ and $\mathrm{W} 4 \mathrm{~N}$ are close to each other in the small and medium aperture ranges, the former is slightly larger than the latter, and the volume dispersion coefficients of $\mathrm{W} 4 \mathrm{~N}$ in the large diameter range 


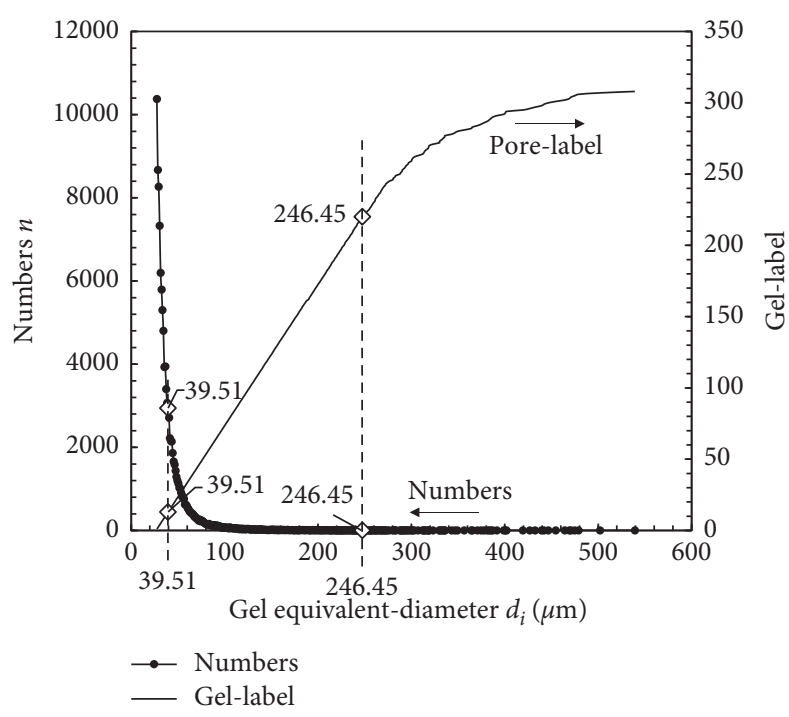

(a)

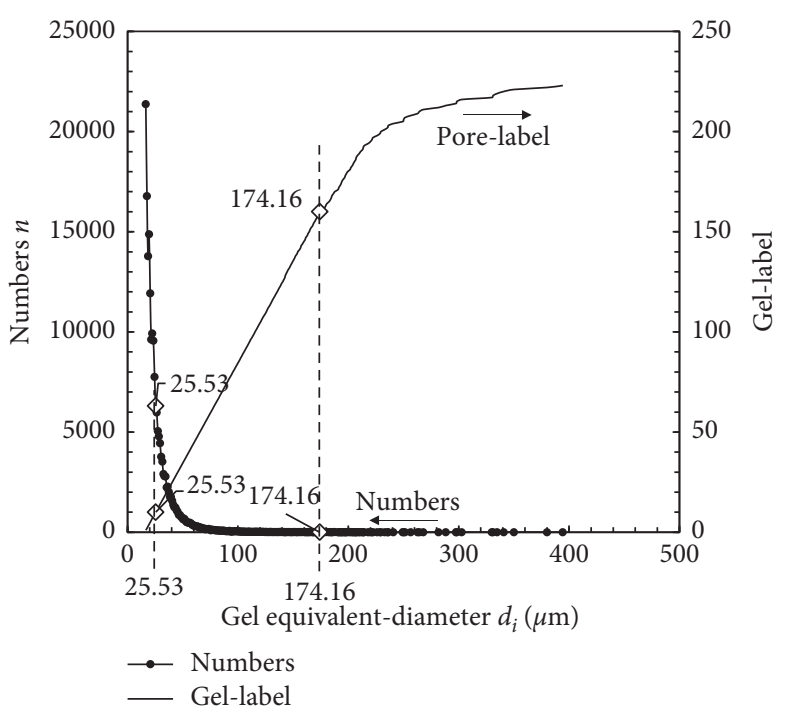

(b)

FIGURE 25: Quantity and continuity distribution of gel-phase of NCG concretion by 3D-XRM. (a) W1 N. (b) W4 N.

are larger than those of $\mathrm{W} 1 \mathrm{~N}$. In the whole diameter distribution interval, the volume dispersion of $\mathrm{W} 1 \mathrm{~N}$ porephase monomer is smaller than that of W4 N.

The quantity proportion of pore-phases and the proportion of the accumulative volume in the above three ranges are shown in Figure 23.

As can be seen from Figure 23, the number of porephases in small diameter range takes up the highest proportion. However, due to the small pore size, the volume takes up the smallest proportion, accounting for about $20 \%$. In the middle diameter range, the number of pore-phases is the second, but the volume proportion is the highest, accounting for about $50 \%$. The number of pore-phases in the large diameter range accounts for the smallest proportion, only about $0.2 \%$, but, due to the large pore size, the volume proportion exceeds that of the small pore size, accounting for about $30 \%$. Through the comparative analysis of the pore-phase number and volume proportion of $\mathrm{W} 1 \mathrm{~N}$ and $\mathrm{W} 4 \mathrm{~N}$ samples, the influence of $\mathrm{W} / \mathrm{C}$ ratio in the three pore diameter ranges is demonstrated (1) In the latter, the quantity proportion of porephase in the small diameter range increases slightly, but the volume proportion remains unchanged, indicating that the pore size in the range decreases. (2) Although the quantity proportion of pore-phase in the latter range decreases slightly, the volume proportion increases, indicating that the pore size increases. (3) The quantity and volume proportion of the latter in the large diameter range are reduced.

The sphericity distributions corresponding to the equivalent-diameter of pore-phase monomer of the two types of concretions and the $\mathrm{C}$. V. of sphericity in three diameter ranges are shown in Figure 24.

It can be seen from Figure 24 that, with the increase of pore-phase equivalent-diameter, the sphericity trend of pore-phase monomer decreases gradually, but the decreasing range of sphericity gradually slows down, and the
C. V. of sphericity is also higher. It indicates that the closer the pore-phase monomer is to the sphere, the more similar and unified the shape characteristics between them are. With the increase of the equivalent-diameter of pore-phase, the difference between the shape of pore-phase monomer and sphere is more and more obvious, and the dispersion and randomness are also higher, and the distribution of shape characteristics is more diverse. The C. V. of the sphericity of the pore-phase monomer of $\mathrm{W} 1 \mathrm{~N}$ and $\mathrm{W} 4 \mathrm{~N}$ were basically similar without obvious difference.

\subsection{Micron-Scale Analysis of Gel-phase of NCG Concretion.} Similar to the analysis mentioned above, the relation curve between the equivalent-diameter of the gel-phase monomer and the gel-label and the quantity of the two types of concretion is given in Figure 25.

As can be seen from Figure 25, the gel-label curve is similar to the pore-label: the monomer diameter in the small diameter range increases uniformly and continuously by a similar amplitude, and the slope of the curve slows down in the later stage, indicating that the monomer diameter of the gel-phase increases by leaps and no longer grows uniformly. The gelphase quantity curve is similar to the pore-phase distribution; that is, the smaller the monomer diameter is, the more the number is, and the distribution is basically in three stages.

By comparing the distribution characteristics of gelphase in Figure 25 with those of pore-phase in Figure 21, we can see that the difference in gel-phase distribution between $\mathrm{W} 1 \mathrm{~N}$ and $\mathrm{W} 4 \mathrm{~N}$ is more significant than that in their porephase distribution, which is mainly reflected in the following two aspects: (1) The pore-phase distribution ranges of the two types of concretions are basically similar, while the gelphase monomer volume, number, and the distribution range of equivalent-diameter of the gel-phase of the two types are significantly different. (2) The segmented feature points of pore-phase number curve of the two types of concretion 
TABLE 5: Interval of equivalent-diameter range of gel-phase by 3D-XRM.

\begin{tabular}{lcccc}
\hline Range & $\begin{array}{c}\text { Small aperture range } \\
\text { Quantity is large and decreases } \\
\text { sharply with diameter }\end{array}$ & $\begin{array}{c}\text { Medium aperture range } \\
\text { Quantity is in the middle and decreases } \\
\text { gradually with diameter }\end{array}$ & $\begin{array}{c}\text { Large aperture range } \\
\text { Quantity is small and does not vary } \\
\text { with diameter }\end{array}$ \\
\hline \multirow{2}{*}{$\begin{array}{l}\text { Type of } \\
\text { concretion }\end{array}$} & W1 & $27.51 \mu \mathrm{m}-39.51 \mu \mathrm{m}$ & $39.51 \mu \mathrm{m}-246.45 \mu \mathrm{m}$ & $246.45 \mu \mathrm{m}-539.25 \mu \mathrm{m}$ \\
& $\mathrm{N} 4$ & $16.59 \mu \mathrm{m}-25.53 \mu \mathrm{m}$ & $25.53 \mu \mathrm{m}-174.16 \mu \mathrm{m}$ & $174.16 \mu \mathrm{m}-394.27 \mu \mathrm{m}$ \\
\hline
\end{tabular}

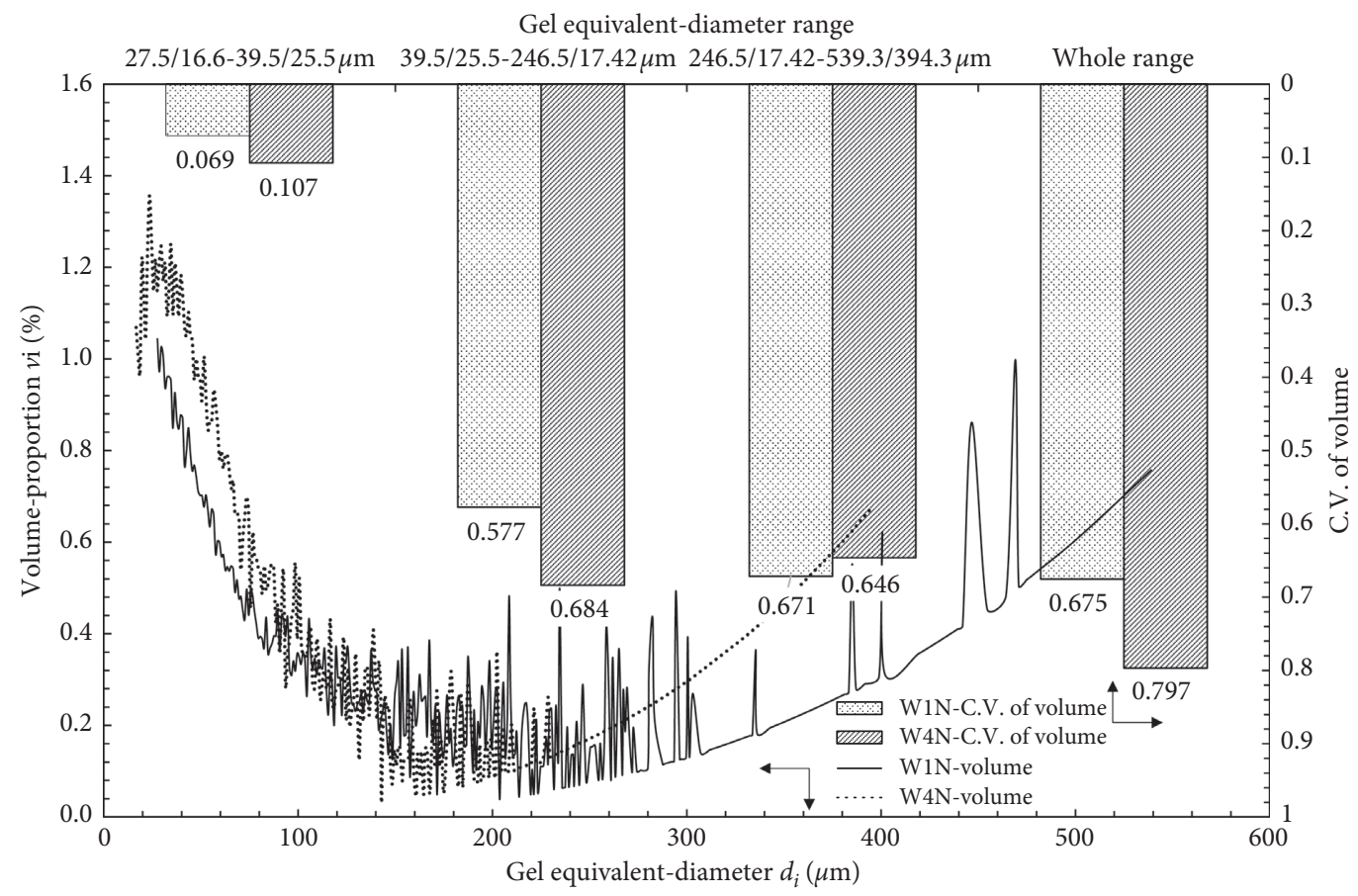

FIgURE 26: Volume distribution and C. V. of volume of gel-phase of NCG concretion by 3D-XRM.

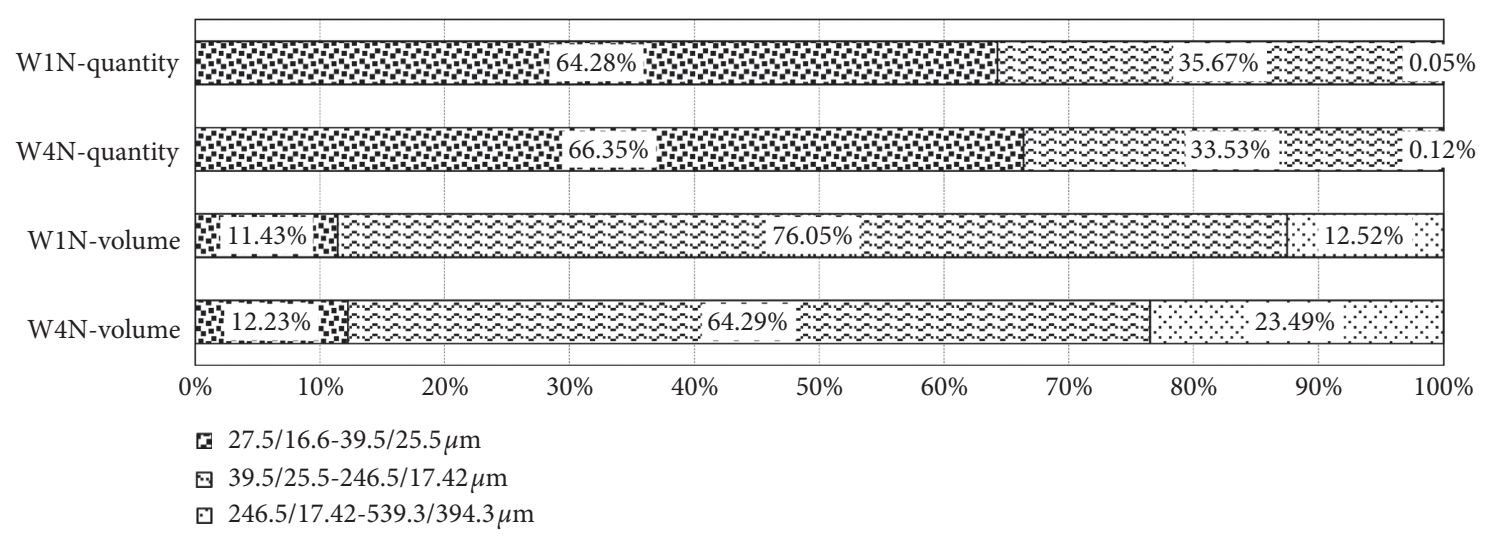

FIGURE 27: Quantity and volume proportion in different gel equivalent-diameter ranges of NCG concretion by 3D-XRM.

were basically similar, while the points of the gel-phase number curve were significantly different (compared with $\mathrm{W} 1 \mathrm{~N}$, the segmented feature points of $\mathrm{W} 4 \mathrm{~N}$ moved forward, and the interval width was also smaller), and the range description was shown in Table 5.
The gel volume distribution of the two types of concretion and the C. V. of the gel-phase monomer volumes in the three diameter ranges are shown in Figure 26.

Figure 26 shows that the volume distribution curve of the gel-phase also shows a trend of first decline and then rise, 


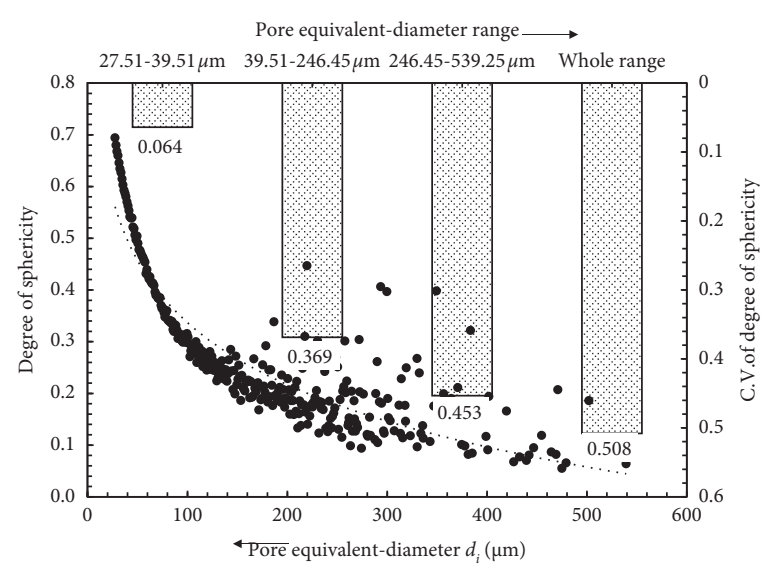

(a)

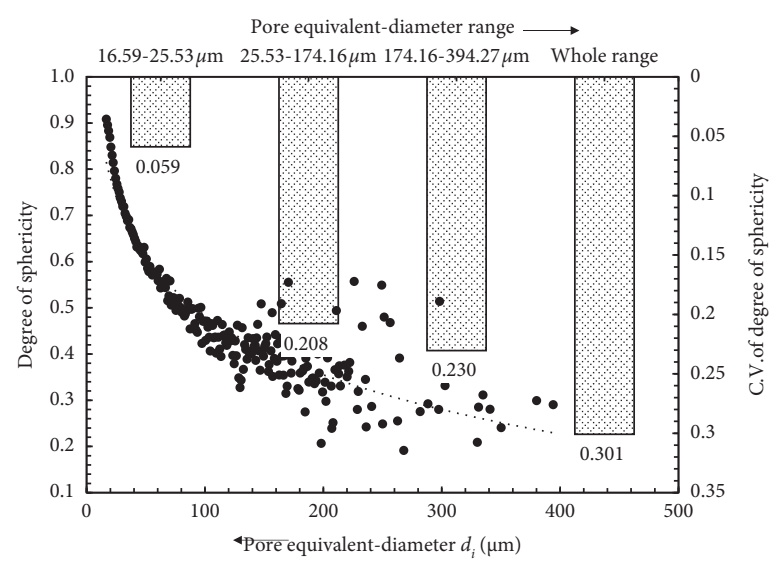

(b)

FIGURE 28: Sphericity distribution and its coefficient of variation of gel-phase of NCG concretion by 3D-XRM. (a) W1 N. (b) W4 N.

which is similar to the pore volume distribution. Compared with the pore volume distribution, the curve of volume distribution curve of gel-phase is more intense, indicating that the number of gel-phase monomers in the adjacent equivalent-diameter interval is quite different, and this difference is reflected in the whole interval. By comparing $\mathrm{W} 1 \mathrm{~N}$ and $\mathrm{W} 4 \mathrm{~N}$, it can be seen that the volume proportion of the latter in the small and large diameter ranges is higher than that of the former, and the difference of gel-phase volume distribution of the two types is higher than that of pore-phase volume distribution. The C. V. of volume in the three ranges of the gel-phase is also significantly different from that of the pore-phase. Among them, the C. V. of volume of the gel-phase of $\mathrm{W} 1 \mathrm{~N}$ still increases with the increase of the diameter. The $\mathrm{C}$. V. of volume in the middle diameter range of $\mathrm{W} 4 \mathrm{~N}$ is the highest, that in the large diameter range is slightly less than that in the middle diameter range, and that in the small diameter range is the lowest. The C. V. of volume of $\mathrm{W} 1 \mathrm{~N}$ in the small and middle diameter ranges are all lower than that of $\mathrm{W} 4 \mathrm{~N}$, while that of $\mathrm{W} 1 \mathrm{~N}$ in the large diameter range is slightly higher than that of $\mathrm{W} 4 \mathrm{~N}$. In all equivalent-diameter ranges, the C. V. of volume of W1 N gel-phase are lower than that of W4 N.

The quantity and volume proportion in the three ranges mentioned above are shown in Figure 27.

As can be seen from Figure 27, the quantity and volume proportion of gel-phases of the two types of concretions are similar to those of pore-phases: (1) The quantity in the small diameter range accounted for the highest proportion, but the volume accounted for the lowest proportion. (2) The quantity proportion in the medium diameter range is in the middle, and the volume proportion is the highest. (3) The quantity proportion in the large diameter range is the lowest, but the volume proportion is the highest. Comparing the volume proportions of pore-phase and gel-phase, the former occupies a more balanced proportion in the three diameter ranges, while the volume of the gel-phase is more concentrated in the medium diameter range, which accounts for a more prominent proportion. Comparing $\mathrm{W} 1 \mathrm{~N}$ and $\mathrm{W} 4 \mathrm{~N}$, with the increase of $\mathrm{W} / \mathrm{C}$ ratio, the quantity and volume proportion in the medium diameter range were reduced, while the proportion in the small diameter range was basically stable, and that in the large diameter range increased.

The sphericity distribution of gel-phase corresponding to equivalent-diameters and the C. V. of sphericity in the three diameter ranges of gel-phase monomers are shown in Figure 28.

It can be seen from Figure 28 that the sphericity of the gel-phase decreases with the increase of the diameter, and the C. V. of sphericity is higher. This indicates that the smaller the diameter of the gel-phase monomer is, the closer the monomer is to the sphere, and the more similar and uniform the shape characteristics are. With the increase of the diameter, the difference between the shape of the gelphase and the sphericity becomes larger and larger, and the higher the dispersion and randomness are, the more diverse the distribution of shape characteristics is. Comparison of $\mathrm{W} 1 \mathrm{~N}$ and $\mathrm{W} 4 \mathrm{~N}$ concretions showed that the difference of sphericity of the gel-phase monomer was significantly higher than that of the pore-phase monomer: the sphericity of the gel-phase monomer of $\mathrm{W} 1 \mathrm{~N}$ was lower than that of $\mathrm{W} 4 \mathrm{~N}$, and the corresponding dispersion coefficient was higher than that of the pore-phase monomer. This indicated that the shape characteristics of the gel-phase monomer of W1 N were more diverse and the shape distribution was more discrete than that of $\mathrm{W} 4 \mathrm{~N}$.

\section{Conclusions}

The following conclusive remarks have been achieved in this research:

(1) NCG material conforms to the $\mathrm{C}_{4} \mathrm{~A}_{3} \overline{\mathrm{S}}-\mathrm{C} \overline{\mathrm{S}}-\mathrm{CaO}-\mathrm{H}_{2} \mathrm{O}$ hydration reaction system, and its solid products are mainly ettringite (AFt), aluminum gel $\left(\mathrm{AH}_{3}\right)$, and calcium silicate hydrate gel $(\mathrm{C}-\mathrm{S}-\mathrm{H})$, showing the characteristics of spatial network structure. With the increase of W/C ratio, AFt crystals become thinner and shorter, and the density and regularity of the network structure also 
decrease. Basic rules of the proportion of solid-phase mass in NCG concretion are as follows: $\mathrm{AFt}$ accounted for the highest proportion, about 65\%; C-S-H accounts for about 20\%; $\mathrm{AH}_{3}$ accounted for the least, about $15 \%$.

(2) The BET adsorption isotherm of NCG concretion belongs to type IV isotherm; characteristics of hysteresis type belong to $\mathrm{H} 3$, with no obvious saturated adsorption platform. It shows the irregular pore structure formed by granular solidphase and fracture. The specific surface area and the MPD of the NCG concretion indicate that the pore types are mainly in mesopore and macropore range.

(3) In nanoscale, NCG concretion is a porous material, and its porosity is more than $60 \%$. With the increase of $\mathrm{W} / \mathrm{C}$ ratio and age, the porosity of concretion increases and the bulk density decreases. With the decrease of $\mathrm{W} / \mathrm{C}$ ratio and the increase of age, the skeleton density (i.e., the density of solid phase) decreases slightly. W/C ratio and age have little influence on solid density of concretion but mainly have significant influence on porosity, thus causing changes in bulk density.

(4) In micron scale, AFt occupies the largest proportion in the NCG concretion body and acts as the main framework. The smaller the $\mathrm{W} / \mathrm{C}$ ratio was, the more uniform and denser AFt was; gel-phase accounted for a larger proportion and pore-phase accounted for a smaller proportion.

(5) In micron scale, the pore-phase and gel-phase characteristics of NCG vary with the diameter in similar ways: With the increase of diameter, the number of monomers gradually decreased and the decreasing rate gradually slowed down. The volume proportion first decreased and then increased, and the difference between the volumes of each monomer gradually increased. The greater the difference between the shapes of monomers and spheres is, the more diverse and discrete the shape characteristics are. The pore-phase and gelphase of NCG concretion are distributed in three stages: (1) small diameter range: the highest quantity proportion and the lowest volume proportion; (2) medium diameter range: the quantity proportion is in the middle, and the volume proportion is the highest; (3) large diameter range: the quantity proportion is the lowest, and the volume proportion is in the middle.

\section{Data Availability}

The data used to support the findings of this study are available from the corresponding author upon request.

\section{Conflicts of Interest}

The authors declare that they have no conflicts of interest.

\section{Acknowledgments}

This work was financially supported by the National Natural Science Foundation of China (Grants nos. 52074270 and 51978657) and the Qing Lan Project of Jiangsu Colleges and Universities of China.

\section{References}

[1] M. R. Della, "Alkali-activated cements opportunities and challenges," Cement and Concrete Research, vol. 29, no. 2, pp. 249-254, 1999.

[2] D. A. Bruce, G. S. Little John, and A. M. C. Naudts, "Grouting materials for ground treatment: a practitioner's guide," ASCE Geotechnical Special Publications, vol. 66, pp. 306-334, 1997.

[3] B. De Paoli, "Fundamental observations on cement based grouts," Traditional Materials Geotechnical Special Publication, vol. 4, pp. 25-28, 1992.

[4] R. W. Boulanger and R. F. Hayden, "Aspects of compaction grouting of liquefiable soil," Journal of Geotechnical Engineering, vol. 12, no. 121, pp. 844-855, 1995.

[5] R. Trauchessec, J.-M. Mechling, A. Lecomte, A. Roux, and B. Le Rolland, "Hydration of ordinary Portland cement and calcium sulfoaluminate cement blends," Cement and Concrete Composites, vol. 56, pp. 106-114, 2015.

[6] S. Allevi, M. Marchi, F. Scotti et al., "Hydration of calcium sulphoaluminate clinker with additions of different calcium sulphate sources," Materials and Structures, vol. 46, no. 1-2, pp. 453-466, 2016.

[7] F. Winnefeld and B. Lothenbach, "Hydration of calcium sulfoaluminate cements - experimental findings and thermodynamic modelling," Cement and Concrete Research, vol. 40, no. 8, pp. 1239-1247, 2010.

[8] M. Zhou, W. Zhang, and B. Li, "Properties of rapid setting and solidifying material with high water content and investigation on its hardening mechanism," Journal of Wuhan University of Technology, vol. 40, no. 8, pp. 18-21, 1998.

[9] H. Xie and C. Liu, "Analysis on influence of moisture content on deformation characteristics of the high-water-content material stone," Journal of Sichuan University (Engineering Science Edition), vol. 44, no. S1, pp. 1-6, 2013.

[10] Y. Jiang, C. Liu, H. Xie et al., "Test and analysis of main physical and mechanical parameters of modified high-waterconcretion," Journal of Sichuan University (Engineering Science Edition), vol. 47, no. S1, pp. 65-69, 2015.

[11] S. Yang, X. Xun, K. Li et al., "Numerical modelling of nonuniform corrosion induced concrete crack width," Journal of Structural Engineering, ASCE, vol. 144, no. 8, Article ID 04018120, 2018.

[12] X. Xun, S. Yang, and C. Q. Li, "A non-uniform corrosion model and meso-scale fracture modelling of concrete," Cement and Concrete Research, vol. 108, pp. 87-102, 2018.

[13] X. Xun and S. Yang, "Investigating the spatial development of corrosion of corner-located steel bar in concrete by $X$-ray computed tomography," Construction and Building Materials, vol. 221, pp. 177-189, 2019.

[14] J. Haas and A. Nonat, "From C-S-H to C-A-S-H: experimental study and thermodynamic modelling," Cement and Concrete Research, vol. 68, pp. 124-138, 2015.

[15] A. M. Cody, H. Lee, R. D. Cody, and P. G. Spry, "The effects of chemical environment on the nucleation, growth, and stability of ettringite [Ca3Al (OH) 6]2 (SO4) 3.26H2O," Cement and Concrete Research, vol. 34, no. 5, pp. 869-881, 2004. 
[16] Y. Jeong, C. W. Hargis, S.-C. Chun, and J. Moon, "The effect of water and gypsum content on strätlingite formation in calcium sulfoaluminate-belite cement pastes," Construction and Building Materials, vol. 166, pp. 712-722, 2018.

[17] Y. Zhang, J. Chang, and J. Ji, "AH3 phase in the hydration product system of AFt-AFm-AH3 in calcium sulfoaluminate cements: a microstructural study," Construction and Building Materials, vol. 167, pp. 587-596, 2018.

[18] K. J. Mun, W. K. Hyoung, C. W. Lee, S. Y. So, and Y. S. Soh, "Basic properties of non-sintering cement using phosphogypsum and waste lime as activator," Construction and Building Materials, vol. 21, no. 6, pp. 1342-1350, 2007.

[19] X. He, C. Miao, J. Hong et al., "Thermal analysis kinetics of cement paste," Journal of Southeast University ( Natural Science Edition), vol. 41, no. 3, pp. 602-605, 2011.

[20] L. Yan, W. Zhou, Y. Qian et al., "Semi quantifying ettringite by DSC-TG method," New Building Materials, vol. 42, no. 2, pp. 41-43, 2015.

[21] International Union of Pure and Applied Chemistry, "Reporting physisorption data forgas/solid systems with special reference to the determination of surface area and porosity," Pure \& Applied Chemistry, vol. 57, no. 4, pp. 603-619, 1985.

[22] S. Brunauer, P. H. Emmett, and E. Teller, "Adsorption of gases in multimolecular layers," Journal of the American Chemical Society, vol. 60, no. 2, pp. 309-319, 1938.

[23] K. Zheng, F. Cai, and X. Zhou, "Study on pore structure of hardened cement-asphalt paste by nitrogen adsorption," Journal of Wuhan University of Technology, vol. 35, no. 2, pp. 1-5, 2013.

[24] E. P. Barrett, L. G. Joyner, and P. P. Halenda, "The determination of pore volume and area distributions in porous substances. I. Computations from nitrogen isotherms," Journal of the American Chemical Society, vol. 73, no. 1, pp. 373-380, 1951.

[25] E. W. Washburn, “The dynamics of capillary flow," Physical Review, vol. 17, no. 3, pp. 273-283, 1921. 